\title{
Galaxy properties as revealed by MaNGA II. Differences in stellar populations of slow and fast rotator ellipticals and dependence on environment
}

\author{
M. Bernardi ${ }^{1 \star}$, H. Domínguez Sánchez ${ }^{1} \dagger$, J. R. Brownstein ${ }^{2}$, N. Drory ${ }^{3}$ and \\ R. K. Sheth ${ }^{1}$ \\ 1 Department of Physics and Astronomy, University of Pennsylvania, Philadelphia, PA 19104, USA \\ 2 Department of Physics and Astronomy, University of Utah, 115 S. 1400 E., Salt Lake City, UT 84112, USA \\ 3 McDonald Observatory, The University of Texas at Austin, 1 University Station, Austin, TX 78712, USA
}

29 August 2019

\begin{abstract}
We present estimates of stellar population (SP) gradients from stacked spectra of slow (SR) and fast (FR) rotator elliptical galaxies from the MaNGA-DR15 survey. We find that: 1) FRs are $\sim 5$ Gyrs younger, more metal rich, less $\alpha$-enhanced and smaller than SRs of the same luminosity $L_{r}$ and central velocity dispersion $\sigma_{0}$. This explains why when one combines SRs and FRs, objects which are small for their $L_{r}$ and $\sigma_{0}$ tend to be younger. Their SP gradients are also different. 2) Ignoring the FR/SR dichotomy leads one to conclude that compact galaxies are older than their larger counterparts of the same mass, even though almost the opposite is true for FRs and SRs individually. 3) SRs with $\sigma_{0} \leq 250 \mathrm{~km} \mathrm{~s}^{-1}$ are remarkably homogeneous within $\sim R_{e}$ : they are old, $\alpha$-enhanced and only slightly super-solar in metallicity. These SRs show no gradients in age and $M_{*} / L_{r}$, negative gradients in metallicity, and slightly positive gradients in $[\alpha / \mathrm{Fe}]$ (the latter are model dependent). SRs with $\sigma_{0} \geq 250 \mathrm{~km} \mathrm{~s}^{-1}$ are slightly younger and more metal rich, contradicting previous work suggesting that age increases with $\sigma_{0}$. They also show larger $M_{*} / L_{r}$ gradients. 4) Self-consistently accounting for $M_{*} / L$ gradients yields $M_{\mathrm{dyn}} \approx M_{*}$ because gradients reduce $M_{\mathrm{dyn}}$ by $\sim 0.2$ dex while only slightly increasing the $M_{*}$ inferred using a Kroupa (not Salpeter) IMF. 5) The FR population all but disappears above $M_{*} \geq 3 \times 10^{11} M_{\odot}$; this is the same scale at which the size-mass correlation and other scaling relations change. Our results support the finding that this is an important mass scale which correlates with the environment and above which mergers matter.
\end{abstract}

Key words: galaxies: structure - methods: observational - surveys

\section{INTRODUCTION}

Hierarchical models seek to understand galaxy formation within the cosmological context of large-scale structure formation (e.g. Mo et al. 2010). These models distinguish between the processes of star formation and its cessation, and those of the assembly of smaller stellar units into what becomes the final object. In these models, there is a close connection between evolution and environment: dense regions are like more evolved universes, so all the action in them

\footnotetext{
* E-mail: bernardm@sas.upenn.edu

$\dagger$ helenado@sas.upenn.edu
}

happened longer ago. Hence, they are expected to be populated by older, more massive galaxies. If these galaxies acquired their stellar mass in situ rather than by a sequence of mergers, then they are expected to show strong gradients in their stellar populations (Larson 1974); gradients which mergers are expected to erase, although residual central star formation can steepen them again (White 1980).

Spatially resolved spectroscopy of large galaxy samples allows one to test such predictions (e.g. Davies et al. 1993). Early work used long slit-spectroscopy to study gradients in small samples of galaxies, finding strong color gradients mostly driven by metallicity (e.g. Sánchez-Blázquez et al. 2007; Spolaor et al. 2009, 2010; Koleva et al. 2011, and references therein). The advent of Integral Field Units (IFUs) 
has driven a revolution in this field (see Cappellari 2016, for a recent review). The SAURON (Emsellem et al. 2004) and ATLAS $^{3 \mathrm{D}}$ (Cappellari et al. 2011) surveys of a decade ago, the more recent CALIFA (Sánchez et al. 2012) and SAMI (Croom et al. 2012) surveys, and the ongoing and substantially larger MaNGA survey (Bundy et al. 2015; Law et al. 2015; Wake et al. 2017; Westfall et al. 2019), provide estimates of kinematic gradients (i.e., rotation curves and velocity dispersion profiles) and stellar population gradients in tens to hundreds of early-type galaxies, each sampled by tens to hundreds of spaxels. There is now general agreement that metallicity increases towards the central regions of early-type galaxies (e.g. Scott et al. 2009; González Delgado et al. 2014; McDermid et al. 2015; González Delgado et al. 2015; Greene et al. 2015; Boardman et al. 2017; van de Sande et al. 2018; Li et al. 2018; Parikh et al. 2019; Zhuang et al. 2019; Zibetti et al. 2019; Ferreras et al. 2019), whereas age gradients are less pronounced. In addition, recent studies of IMF gradients (Martín-Navarro et al. 2015a; MartínNavarro et al. 2015c,b; La Barbera et al. 2016; Vaughan et al. 2018a,b; Sarzi et al. 2018) consistently find that the central regions of galaxies favour a bottom-heavy IMF.

Most of these studies are based on samples that are significantly smaller than what MaNGA provides. The larger sample size, and the availability of more precise morphological classifications (Fischer et al. 2019), motivated us (Domínguez Sánchez et al. 2019, hereafter Paper I) to divide MaNGA early-types into Ellipticals (Es) and S0s, and study gradients in the Es. (The S0s are studied in Paper III - in prep.) In particular, in Paper I we constructed stacked spectra of Es at $z<0.08$ binned in $\sigma_{0}$ and luminosity $L_{r}$ using the multiple spectra provided by the MaNGA survey (Bundy et al. 2015) which is a component of the Sloan Digital Sky Survey IV (Blanton et al. 2017). We estimated absorption line strengths in these high $\mathrm{S} / \mathrm{N}$ spectra, and then used a variety of single stellar population synthesis models to estimate stellar population parameters. We focused on the effects of the IMF variations inside a galaxy and across the galaxy population. We found that for the ellipticals with the largest $L_{r}$ and $\sigma_{0}$, the results are consistent with those associated with the commonly used Salpeter IMF in the central regions, approaching values from a Kroupa-like IMF by $\sim 1 R_{e}$ (assuming $[\mathrm{X} / \mathrm{Fe}]$ enhancement variations are limited). For these galaxies we find that the stellar mass-tolight ratio decreases at most by a factor of 2 from the central regions to $R_{e}$. In contrast, for lower $L_{r}$ and $\sigma_{0}$ galaxies, the IMF is shallower and the $M_{*} / L_{r}$ of central regions is similar to the outskirts. That gradients become less important at lower masses is also consistent with previous work (Parikh et al. 2018). Although a factor of 2 is smaller than previous reports based on a handful of galaxies (e.g. van Dokkum et al. 2017), it is still large enough to matter for dynamical mass estimates (Bernardi et al. 2018b). Our results show that accounting self-consistently for the $M_{*} / L_{r}$ gradients when estimating both $M_{*}$ and $M_{\text {dyn }}$ brings the two into good agreement: gradients reduce $M_{\text {dyn }}$ by $\sim 0.2$ dex while only slightly increasing the $M_{*}$ inferred using a Kroupa IMF. This is a different resolution of the $M_{*}-M_{\mathrm{dyn}}$ discrepancy than has been followed in the recent literature where $M_{*}$ of massive galaxies is increased by adopting a Salpeter IMF while leaving $M_{\text {dyn }}$ unchanged (e.g. Cappellari et al. 2013; Li et al. 2017). Our results are consistent with previous work on reconciling estimates of the stellar and dynamical mass functions (Bernardi et al. 2018b).

Paper I also found that galaxies with larger $\sigma_{0}$ or $L_{r}$ tend to be older and more metal rich, and that, within a galaxy, age and metallicity tend to increase with $\sigma$ : i.e., they are all larger in the center. These findings are in qualitative agreement with previous work (e.g. La Barbera et al. 2013; Martín-Navarro et al. 2015a; La Barbera et al. 2016; van Dokkum et al. 2017; Tang \& Worthey 2017; Parikh et al. 2018). However, in Paper I we also found that Es with $200 \leq$ $\sigma_{0} \leq 250 \mathrm{~km} \mathrm{~s}^{-1}$ and $-21.5 \leq M_{r} \leq-22.5$ tend to be the oldest in the sample, even though they are neither the largest $\sigma$ nor the largest $L_{r}$. One of the goals of the present study is to investigate why.

In addition, recent work has emphasized the fact that, if early-type galaxies are classified as being slow or fast rotators (following Emsellem et al. 2007), then the slow rotators are much more likely to have had merger-dominated assembly histories (Cappellari 2016). Fischer et al. (2019) showed the value of using the Domínguez Sánchez et al. (2018) morphological classificiations to separate the Es from S0s in MaNGA when studying the slow and fast rotator dichotomy. This motivates us to further subdivide our sample of Es on this basis. Additional motivation comes from the fact that McDermid et al. (2015) do not report a significant difference in the age of the stellar populations of slow and fast rotating early-type galaxies in the ATLAS ${ }^{3 \mathrm{D}}$ survey (they only find a significant difference in the metallicity, with fast rotators being more metal rich), whereas van de Sande et al. (2018) find that fast rotators in the SAMI survey tend to be younger. Our MaNGA sample should allow us to determine whether or not rotation plays a key role and, if it does, whether the age-rotation anti-correlation is driven by morphology (e.g. S0s are fast and Es are slow rotators) or is present even in a sample of Es alone. ${ }^{1}$ McDermid et al. (2015) do report another correlation with age: At a given mass, more compact galaxies are older. We study if this anti-correlation between age and size is present in our sample, although it spans a much smaller range of $L$ and $\sigma_{0}$, and how it relates to the slow/fast rotator dichotomy.

In Section 2 we first subdivide each $L_{r}$ and $\sigma_{0}$ bin on the basis of half-light radius $R_{e}$, and then still further on the basis of rotation. Section 3 provides estimates of the stellar populations (age, metallicity, $\alpha$-enhancement, IMF and stellar mass-to-light ratio) and stellar population gradients in these bins. It also discusses the ages and sizes at a given $M_{*}$ accounting for the slow/fast rotator dichotomy and compares stellar and dynamical mass estimates of slow and fast rotators when one self-consistently accounts for $M_{*} / L$ gradients. Section 4 discusses how the observed properties correlate with environment. A final section summarizes. Appendix A demonstrates that, even after subdividing by size

1 If most fast rotating Es are simply mis-classified S0s (e.g. Emsellem et al. 2011; Cappellari 2016) then the issue of morphology dependence is less interesting. However, because there exists substantial previous work on S0s as opposed to Es in MaNGA (Fraser-McKelvie et al. 2018, and references therein), we thought it useful to keep the two populations separate. Moreover, it may be that stellar population gradients, which were not the focus of the ATLAS ${ }^{3 \mathrm{D}}$ or SAMI analyses, are able to discriminate between S0s and fast rotators Es. 


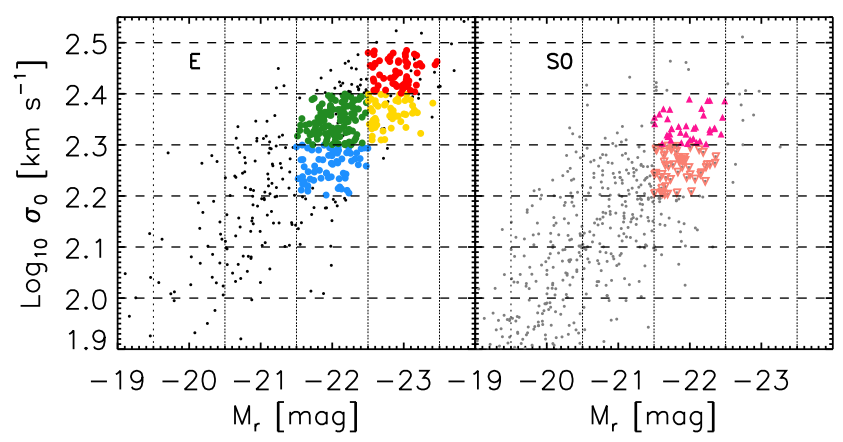

Figure 1. Correlation between $\sigma_{0}$ and $L_{r}$ for Es (left) and S0s (right) at $z \leq 0.08$ selected as described in Paper I. Blue, green, yellow and red symbols in the left-hand panel show Es in bins B00, B10, B11 and B21 (see Table 1). Open inverted and filled upright triangles in the right-hand panel show S0s in bins B00 and B10; there are essentially no S0s in bins B11 and B21.

and rotation, our stacked spectra have sufficient $\mathrm{S} / \mathrm{N}$ to provide reliable measurements. It also discusses how, when studing gradients, one must be careful about the fact that, if the same galaxy is located closer to us, then its inner regions will be sampled by more MANGA spaxels, but its outer regions may not be sampled at all. Appendix B contrasts our results with previous work.

\section{BINNING IN $\sigma_{0}, L_{R}, R_{E}$ AND ROTATION}

In Paper I we defined a sample of MaNGA Es with redshift $z \leq 0.08$ to cover only a small range of lookback times. We constructed stacked spectra of these MaNGA Es, binned in $\sigma_{0}$ and luminosity $L_{r}$. Here we further subdivide each bin into objects with above and below average sizes $R_{e}$ within the bin, before making stacked spectra. (In what follows $R_{e}$ is the truncated semimajor axis, i.e. $R_{e}=R_{e, \text { maj }}$, from the best-fit indicated by FLAG_FIT in the MaNGA PyMorph Photometric Value Added Catalogue, hereafter MPP-VAC, see Fischer et al. 2019). We then separate slow and fast rotators in each $\sigma_{0}, L_{r}$ and $R_{e}$ bin.

Although the results which follow are all based on splitting the sample based on the distribution in the $R_{e}-L_{r}$ plane at each $\sigma_{0}$, we have also studied what happens if we split based on the $R_{e}-\sigma_{0}$ plane at each $L_{r}$. This results in similar, but noisier trends, compared to those we show below. We comment on splitting based on Fundamental Plane parameters $R_{e}, \sigma_{0}$ and surface-brightness in Appendix B.

\subsection{Splitting on size at fixed $\sigma_{0}$ and $L_{r}$}

The left hand panel of Figure 1 shows how Paper I defined the $\sigma_{0}$ and $L_{r}$ bins we use in our study of Es. Table 1 describes the bin limits, and the number of objects in each bin. Note that bins B00 and B10 have the same $L_{r}$ but different $\sigma_{0}$, as do bins $\mathrm{B} 11$ and $\mathrm{B} 21$; bins $\mathrm{B} 10$ and $\mathrm{B} 11$ have the same $\sigma_{0}$ but different $L_{r}$. One of our tests will compare these Es with S0s. Therefore, the right hand panel shows the S0 distribution in the corresponding bins. Notice that there are essentially no S0s brighter than $M_{r} \leq-22.5$. It is worth noting that this is the luminosity scale (mass scale $M_{*} \sim 2 \times 10^{11} M_{\odot}$ assuming a Chabrier IMF) at which many



Figure 2. Size-luminosity relation of MaNGA elliptical galaxies in the four bins described in Table 1. These were further subdivided into two approximately equal bins in size using the four straight lines shown. Lighter shades (light blue, light green, orange and pink) represent galaxies with smaller sizes given their $\sigma$ and $L_{r}$; darker shades (dark blue, dark green, brown, red) represent galaxies with larger sizes. Triangles show S0s in the bin B00 and B10 (i.e., compare with the blue and green Es, respectively).

BINNING OF GALAXIES

\begin{tabular}{lcccccc}
\hline Bin & $\begin{array}{c}\mathrm{M}_{r} \\
{[\mathrm{mag}]}\end{array}$ & $\begin{array}{c}\log _{10} \sigma_{0} \\
{\left[\mathrm{~km} \mathrm{~s}^{-1}\right]}\end{array}$ & All & SR & $\begin{array}{c}\text { FR } \\
\lambda_{e}<0.2\end{array}$ & $\begin{array}{c}\text { FR } \\
\lambda_{e}>0.2\end{array}$ \\
\hline B00-S & $-21.5,-22.5$ & $2.20,2.30$ & 33 & 5 & 6 & 22 \\
B00-L & $-21.5,-22.5$ & $2.20,2.30$ & 37 & 20 & 9 & 8 \\
B10-S & $-21.5,-22.5$ & $2.30,2.40$ & 64 & 25 & 18 & 21 \\
B10-L & $-21.5,-22.5$ & $2.30,2.40$ & 57 & 27 & 14 & 16 \\
B11-S & $-22.5,-23.5$ & $2.30,2.40$ & 28 & 13 & 2 & 13 \\
B11-L & $-22.5,-23.5$ & $2.30,2.40$ & 24 & 13 & 7 & 4 \\
B21-S & $-22.5,-23.5$ & $2.40,2.50$ & 28 & 16 & 9 & 3 \\
B21-L & $-22.5,-23.5$ & $2.40,2.50$ & 32 & 18 & 8 & 6 \\
\hline \hline
\end{tabular}

Table 1. Number of galaxies in each bin with large $R_{e}\left(\mathrm{~B}^{* *}-\mathrm{L}\right)$ and small $R_{e}\left(\mathrm{~B}^{* *}-\mathrm{S}\right)$ with respect to the mean $R_{e}-L_{r}$ relation. Slow (SR) and fast (FR) rotators are galaxies inside and outside the box in the bottom corner of Figure 4, respectively.

early-type galaxy scaling relations change slope (Bernardi et al. 2011), and where the population becomes dominated by slow rotators (Cappellari 2016). In Section 3.5 we show that the IMF is not Chabrier, so we provide a better estimate of this critical stellar mass scale in 3.7.

Figure 2 illustrates how the galaxies in each $\sigma_{0}$ and $L_{r}$ bin were subdivided based on $R_{e}$. Note that the division is not a straight cut on $R_{e}$; rather we separate the objects in a bin based on whether they are larger or smaller than the mean $R_{e}-L_{r}$ relation for the bin. Hereafter, we use B00-L and B00-S to refer to the objects in bin B00 that are larger or smaller than the average for their $L_{r}$ (and $\sigma_{0}$ ), and similarly for the other bins. On average, the objects in $\mathrm{B}^{* *}-\mathrm{L}$ have smaller surface brightnesses than those in $\mathrm{B}^{* *}$-S. In the next subsection (Section 2.2) we subdivide even further based on angular momentum: Table 1 gives the relevant numbers for this as well. 


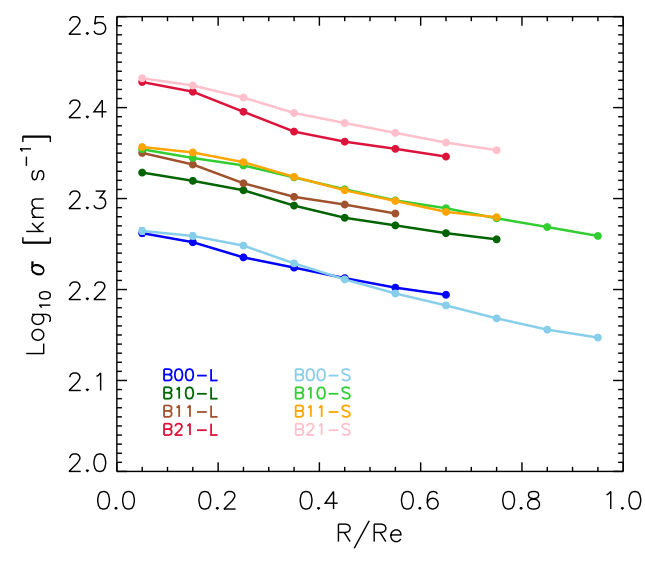

Figure 3. Velocity dispersion profiles for the eight bins described in Table 1. Except for bin B00 (blue), the objects with larger sizes (for their central velocity dispersion and $L_{r}$ ) in a bin tend to have $\sigma$ at $R / R_{e} \sim 0.5$ smaller by about 0.02 dex.

We cannot subdivide into arbitrarily fine bins, since we require spectra with signal-to-noise $\sim 100$ and this is larger than that in a typical spaxel (Figure A1 in Appendix A). To produce high signal-to-noise spectra, spaxels of the galaxies in each of the bins defined in Table 1 were stacked based on their values of $R / R_{e}$ (see Paper I for details). The number of spaxels which contribute, and the resulting $\mathrm{S} / \mathrm{N}$ ratios are provided in Appendix A. In particular, Figure A2 shows the number of spaxels in each radial bin which contribute to our results for the eight bins in $L_{r}, \sigma_{0}$ and $R_{e}$. Some of the curves decrease at large $R$ because, for the largest galaxies, the spaxels may not cover the entire region within $R_{e}$. Figure A2 shows that this problem is most severe for bin B11-L. In what follows we only show those radial bins which are well represented by all galaxies (e.g. for bin B11-L (brown) we show our results out to $R / R_{e} \sim 0.6$ and for B21$\mathrm{L}$ (dark red) out to $\left.R / R_{e} \sim 0.7\right)$. Moreover, within a bin, we expect some galaxy-to-galaxy variation over which we would like to average so as to obtain representative values. Therefore, we also require more than $\sim 10$ galaxies per stack. While this is not an issue for the analysis of this Section, we must take care when we subdivide based on rotation in the next Section.

Figure 3 shows the velocity dispersion profiles for these bins. While the binning was based on $L_{r}$ and the central $\sigma_{0}$, the bins with larger $R_{e}$ in each bin tend to have slightly smaller velocity dispersions at larger $R$. Nevertheless, it is apparent that $\sigma_{0}$ of galaxies in bin B00 is approximately the same as $\sigma_{e}$ at the outer regions $\left(\sim R_{e}\right)$ of bins $\mathrm{B} 10$ and $\mathrm{B} 11$. Similarly, $\sigma_{0}$ of B10 and B11 galaxies is similar to $\sigma_{e}$ of B21 galaxies. This will allow us to test if stellar population parameters which vary with $\sigma$ across the population are really a function of the local $\sigma$.

\subsection{Separating fast and slow rotators}

We noted in the introduction that it is interesting to further subdivide our sample on the basis of rotation, in part because slow rotators are expected to have merger-dominated histories.

Figure 4 shows the joint distribution of photometric ellipticity $\epsilon \equiv 1-b / a$ and $\operatorname{spin} \lambda_{e} \equiv$

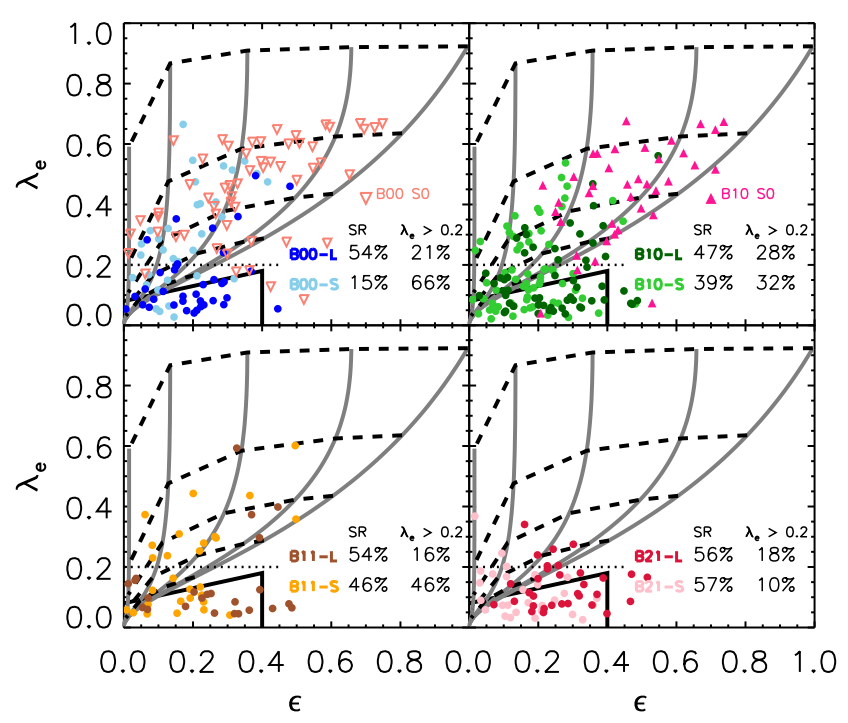

Figure 4. Joint distribution of shape $\epsilon \equiv 1-b / a$ and $\operatorname{spin} \lambda_{e}$ (defined in the main text) for the objects (filled circles) in our four bins. The open and filled triangles show the S0s (there are essentially no S0s in bins B11 and B21). Right-most solid grey line shows an isotropic rotator viewed edge-on; the others show different inclinations. Dashed lines show how an object of intrinsic $\epsilon$ (given by the right-most value) moves across the diagram as inclination changes. Black box in bottom left corner of each panel shows the region which defines 'slow rotators'. In each bin, the objects with larger $R_{e}\left(\mathrm{~B}^{* *}-\mathrm{L}\right)$ always have a significantly larger fraction of 'slow rotators' (approximately the same for each bin), while for galaxies with smaller $R_{e}\left(\mathrm{~B}^{* *}-\mathrm{S}\right)$ large $\lambda_{e}$ values are more common in the top left panel (bin B00).

$\sum_{i} F_{i} R_{i}\left|V_{i}\right| / \sum_{i} F_{i} R_{i} \sqrt{V_{i}^{2}+\sigma_{i}^{2}}$, where $F$ is the flux, $V_{i}$ and $\sigma_{i}$ are the rotation speed and velocity dispersion in pixel $i$ which is at distance $R_{i}$ from the image center, and the sum is over all spaxels within $R_{e}$ (Emsellem et al. 2007). (We have corrected this quantity for seeing following Graham et al. 2018.) The box in the bottom corner shows the region which is used to identify slow rotators (hereafter SR; see, e.g., Cappellari 2016). Objects outside this box are usually called fast rotators (FR). In what follows, we will sometimes select objects with $\lambda \geq 0.2$ as a simple way of decreasing contamination from SRs, and will often simply refer to them as FRs. The text in each panel shows the fraction of FRs and those with $\lambda_{e}>0.2$ in each bin, and Table 1 gives the actual number of SRs, FRs with $\lambda_{e}<0.2$ and FRs with $\lambda_{e}>0.2$ in each bin.

Graham et al. (2018) and Fischer et al. (2019) show how MaNGA galaxies populate this plane as a function of morphology. Here, we mainly concentrate on Es, and further subdivide the Es based on $L_{r}, \sigma_{0}$ and $R_{e}$. It is clear that large $\lambda_{e}$ values are more common in the top left panel (bin B00) than bottom right (B21). It is well known that SRs only begin to dominate at large $L_{r}$ and that the typical $\lambda_{e}$ for Es decreases as $L_{r}$ increases (Cappellari 2016; Fischer et al. 2019). Note however that the SR fraction is approximately independent of $L_{r}$ for the larger objects $\left(\mathrm{B}^{* *}-\mathrm{L}\right)$ in each $\sigma_{0}$ and $L_{r}$ bin.

Finally, consider the open inverted and filled upright triangles in the two top panels. These show S0s with the same $L_{r}$ and $\sigma_{0}$ in each bin. (It is worth noting that the $\mathrm{S} 0$ vs E morphological classification was based entirely on imaging: 


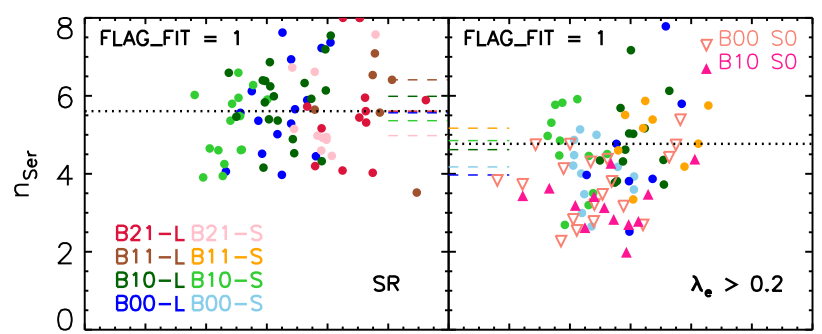

$\begin{array}{lllllllllllllll}0.2 & 0.4 & 0.6 & 0.8 & 1.0 & 1.2 & 0.2 & 0.4 & 0.6 & 0.8 & 1.0 & 1.2 & 1.4\end{array}$ $\log _{10} R_{e-m a j}[k p c] \quad \log _{10} R_{e-\text { maj }}[k p c]$

Figure 5. Correlation between Sersic index $n$ and size $R_{e}$ in our eight bins, for SRs (left) and FRs (right) with FLAG_FIT=1 (i.e. objects whose photometry is better described by a single Sersic profile: $\sim 64 \%$ of our Es). Dotted horizontal lines show the median $n$ for the Es in each panel. Dashed colored horizontal lines show the median $n$ in each bin: SRs tend to have larger $n$ than their FR counterparts. Triangles in the right hand panel show FLAG_FIT $=1$ S0s ( $\sim 35 \%$ of the S0s) with the same $L_{r}$ and $\sigma_{0}$ as the Es in bins B00 and B10; they have substantially lower $n$.

i.e. it did not use $\lambda_{e}$ at all.) Clearly, the S0s are generally rotating faster than the Es. In addition, while we see S0s with $\epsilon<0.2$ in bin B00 (triangles in the top left panel) there are no S0s with $\epsilon \leq 0.2$ in bin B10 (filled triangles in top right panel). We will discuss this in the next Section.

\subsection{Separating Es from S0s}

Figure 5 shows additional evidence of an important structural difference between Es and S0s: the correlation between Sersic index $n$ and size $R_{e}$ in our eight bins, for SRs (left) and FRs (right). Here we show galaxies with FLAG_FIT=1 ( $\sim 64 \%$ of our E sample), i.e. whose photometry is better described by a single Sersic profile (see Fischer et al. 2019 for details). We also only show those bins which include at least five galaxies. The dotted horizontal lines show the me$\operatorname{dian} n$ for the Es in each panel, and dashed lines show the median for the Es in each bin. This shows that SR Es tend to have larger $n$ than their FR counterparts. The open and filled triangles in the FR panel show that the B00 and B10 S0s $(\sim 35 \%$ of the S0s have FLAG_FIT=1) tend to have the smallest $n$ at each $R_{e}$ than their fast rotating $\mathrm{E}$ counterparts.

To study the other $36 \%$ of our E sample ( 65\% for S0s), Figure 6 shows the objects classified as having FLAG_FIT $=0$ or 2 (as for the previous Figure, we only show those bins which include at least five galaxies). These are objects for which a two-component Sersic+Exponential fit was as good as or preferred to the single-Sersic fit. In this case, rather than showing $n$ of the Sersic bulge component we show $\mathrm{B} / \mathrm{T}$, the fraction of the light that is associated with the bulge. The panel on the left shows that although some of the slow rotator Es may have two components, they always have $\mathrm{B} / \mathrm{T}>0.6$ or so. Note that there are no dark or light blue symbols in this panel: almost none of the SR Es in bin B00 are 2-component systems. The panel on the right shows fast rotators. Here, there are no dark blue or green symbols - i.e., most of the larger galaxies in a bin are well described by a single component. While some of the more compact Es in a bin (light blue and light green) are better fit by two components, these tend to have $\mathrm{B} / \mathrm{T}$ values that



Figure 6. Correlation between bulge to total fraction $\mathrm{B} / \mathrm{T}$ and size $R_{e}$ in our eight bins, for SRs (left) and FRs (right) with FLAG_FIT $=0$ or 2 (i.e. objects whose photometry is as well or better-fit by a two-component Sersic+Exponential profile: $\sim 36 \%$ of our Es). Dotted horizontal lines show the median B/T for the Es in each panel. Dashed colored horizontal lines show the median $\mathrm{B} / \mathrm{T}$ in each bin. Triangles in the right hand panel show FLAG_FIT $=0$ or 2 SOs $(\sim 65 \%$ of the S0s $)$ with the same $L_{r}$ and $\sigma_{0}$ as the Es in bins B00 and B10; they have lower B/T.

are similar to the SRs. In particular, these $\mathrm{B} / \mathrm{T}$ values are larger than for the S0s of similar $L_{r}$ and $\sigma_{0}$.

Although S0s tend to have the smallest $n$ and $\mathrm{B} / \mathrm{T}$ at each $R_{e}$ compared to their fast rotating $\mathrm{E}$ counterparts, in neither case is there an obvious gap between the FRs Es and S0s. Also there are no S0s with ellipticity $\epsilon \leq 0.2$ in bin $\mathrm{B} 10$ of Figure 4 (filled triangles in top right panel). Since the dashed lines trace the expected locus in the $\lambda_{e^{-}} \epsilon$ plane as the inclination with respect to the line of sight changes, seeing objects at large $\epsilon$ but not at small values may indicate that our Deep Learning morphologies have a tendency to label face-on S0s as Es. This is less of an issue for bin B00 where we do see S0s with $\epsilon<0.2$ (triangles in the top left panel of Figure 4), so if there is an inclination-dependent bias, it is $\sigma_{0}$-dependent. Fischer et al. (2019) provide more discussion of the $\epsilon$ distribution. In addition, the majority of Es are clearly single component systems, whereas the majority of S0s are clearly not and (although we do not show it here) the average $\mathrm{B} / \mathrm{T}$ of $\mathrm{S} 0 \mathrm{~s}(\sim 0.4)$ is smaller than that of Es $(\sim 0.6)$ even at smaller ellipticity. So as to reduce the question of morphological effects as much as possible, we have chosen to separate FR Es from S0s in the analysis which follows. The next section, where we consider stellar populations, will show if this was necessary. (One can always perform a weighted sum of the E and S0 results should the separation prove to have been unnecessary.)

\subsection{Correlation between size and rotation for Es}

Since dissipationless mergers are expected to increase $n$ (e.g. Hilz et al. 2013), it is tempting to view the differences between the Es in the two panels of Figure 5 as further evidence that SR Es had merger-dominated histories. Dissipationless mergers are also expected to increase galaxy sizes more dramatically than velocity dispersion. So it is interesting that SRs tend to lie slightly above the $R_{e}-L_{r}$ relation defined by all the objects in the bin, whereas FRs tend to lie slightly below it (compare left and right hand panels of Figure 7). However, the scatter around the bin-dependent $R_{e}-L_{r}$ relation decreases as $L_{r}$ increases (Figure 8) and it is not obvious that mergers can explain a decrease in this scatter. Never- 




Figure 7. Residuals with respect the mean relation for each bin (i.e. the solid lines in Figure 2) of the size-luminosity distribution for slow (left) and fast (right) rotators. Dotted horizontal lines show the median residual for the Es in each panel, and dashed horizontal lines in each panel show the median residual in each bin: SRs are slightly larger than FRs in essentially all bins.



Figure 8. Scatter around the $R_{e}-L_{r}$ relation decreases as $L_{r}$ increases. For slow rotators (left), this scatter is slightly smaller for the smaller objects in a given $\left(\sigma_{0}, L_{r}\right)$ bin. Here, we only show those bins which include enough galaxies to draw statistically significant conclusions (see text).

theless, the fact that SRs tend to be larger than FRs means that one must be careful to separate correlations with size from those with rotation. The results of the next section illustrate this nicely.

While we have pointed out a number of circumstantial lines of evidence linking SRs to mergers, the next section provides a more careful consideration of the stellar populations. As we remarked earlier, this requires that we stack spectra, which, in turn is limited by the number of objects in a bin. This number is provided in Table 1. If it is less than 10, then we do not use the bin for subsequent analysis, on the grounds that we cannot be certain of drawing statistically significant conclusions. In addition, as we remarked earlier, we also must be certain that these objects contribute enough spaxels at each $R$ with which to make a stack of sufficiently high S/N. Figure A5, the analog of Figure A2, shows the number of spaxels in each radial bin which contribute to our SR and FR results for the eight $L_{r}, \sigma_{0}$ and $R_{e}$ bins. The results which follow are based only on scales indicated by solid lines in Figure A5. Dotted lines indicate too few galaxies in the bin (e.g. there are not enough objects with $\lambda_{e} \geq 0.2$ in bins B00-L, B11-L, B21-L and B21-S, and not enough SRs in bin B00-S), and dashed lines indicate scales which are compromised by the fact that the spaxels may not cover the entire region within $R_{e}$ (so the number of spaxels is decreasing rather than increasing with $R$ ). This matters mainly for SRs in bins B00-L, B10-L, B21-L and especially B11-L.

\section{SINGLE STELLAR POPULATION PARAMETERS}

We now describe differences between the stellar populations in the bins defined in Table 1. We first present measurements of a number of spectral features, and then the result of using single stellar population synthesis models (SSPs) to interpret these measurements in terms of IMF shape, age, metallicity $[\mathrm{M} / \mathrm{H}], \alpha$-element abundance $[\alpha / \mathrm{Fe}]$ and stellar mass-to-light ratio $M_{*} / L_{r}$. We study how SSP results depend on size and rotation, both as a function of the local value of $\sigma$ and galactocentric distance $R$.

Paper I compared a variety of single SP synthesis models, finding differences in overall values, but much smaller differences regarding relative comparisons. E.g. whereas different SSPs might disagree on the value of the age of the stellar population, if one SSP finds that two bins differ in age by $\sim 2$ Gyrs, then the other is likely to also. ${ }^{2}$ Therefore, in what follows, we only show results based on the MILES-library with Padova isochrones and BiModal IMFs (Vazdekis et al. 2015). In these models, the scaled-solar spectra (i.e. $[\alpha / \mathrm{Fe}]=0$ ) have abundances from Grevesse \& Sauval (1998), whereas the $\alpha$-enhanced spectra $([\alpha / \mathrm{Fe}]=+0.4)$ assume that $[\mathrm{X} / \mathrm{Fe}]=+0.4$ for the elements $\mathrm{O}, \mathrm{Ne}, \mathrm{Mg}, \mathrm{Si}$, $\mathrm{S}, \mathrm{Ca}$ and $\mathrm{Ti}$, and that the other elements have solar abundances. Note that, since we only use a combination of Fe and $\mathrm{Mg}$ lines to constrain the $\alpha$-enhancement (see middle panel in Figure 9) without taking individual element ratios into account, in practice the $[\alpha / \mathrm{Fe}]$ we report is based entirely on the $[\mathrm{Mg} / \mathrm{Fe}]$ abundance (e.g. Johansson et al. 2012).

\subsection{Lick index diagnostics}

We measured a number of Lick indices in the stacked spectra $\left(\mathrm{H}_{\beta}, \mathrm{Mg} b, \mathrm{Fe} 5270, \mathrm{Fe} 5335, \mathrm{TiO} 2_{\mathrm{SDSS}}\right.$; see Table 3 of Paper I for details, where we also describe how the two Fe lines are combined to make what is refered to as $\langle\mathrm{Fe}\rangle$, and how they are combined with $\mathrm{Mg} b$ to make $[\mathrm{MgFe}])$.

Figure 9 shows the $\mathrm{H}_{\beta}-[\mathrm{MgFe}],<\mathrm{Fe}>-[\mathrm{MgFe}]$ and $\mathrm{TiO}_{\mathrm{SDSS}^{-}}[\mathrm{MgFe}]$ diagnostic plots. The first is an indicator of ages and metallicities; the second of $\alpha$-element abundances; and the third is sensitive to the IMF. The objects with smaller sizes (lighter shades: light blue, light green, orange, pink) clearly have stronger $\mathrm{H}_{\beta}$ and, except for bin B00, stronger $<\mathrm{Fe}>$ and weaker $\mathrm{TiO} 2_{\mathrm{SDSS}}$. Superimposed on the measurements are model grids corresponding to a bimodal IMF with slope 2.3 and the assumed value of $[\alpha / \mathrm{Fe}]$ shown.

Before we use SSPs to interpret these differences, Figures 10 and 11 show the $\mathrm{H}_{\beta}$-[MgFe] and $<\mathrm{Fe}>-[\mathrm{MgFe}]$ distributions for the SR and FRs in each bin. (There are not enough FRs in bin B21, so we only show results for the other 3 bins.) Each panel shows four sets of symbols, corresponding to the separation based on size (light vs dark shades), further subdivided into SRs and FRs (crosses and ellipses) where possible: For B00-L only SRs are shown (blue); for B00-S only FRs (light-blue); and for B11-L only SRs (brown). Filled circles (without error bars) show the

2 It is worth noting explicitly that all the ages we quote are $\mathrm{H}_{\beta}$ luminosity weighted ages. Recent star formation by a small fraction of the population will bias these ages younger than 'massweighted' ages. 

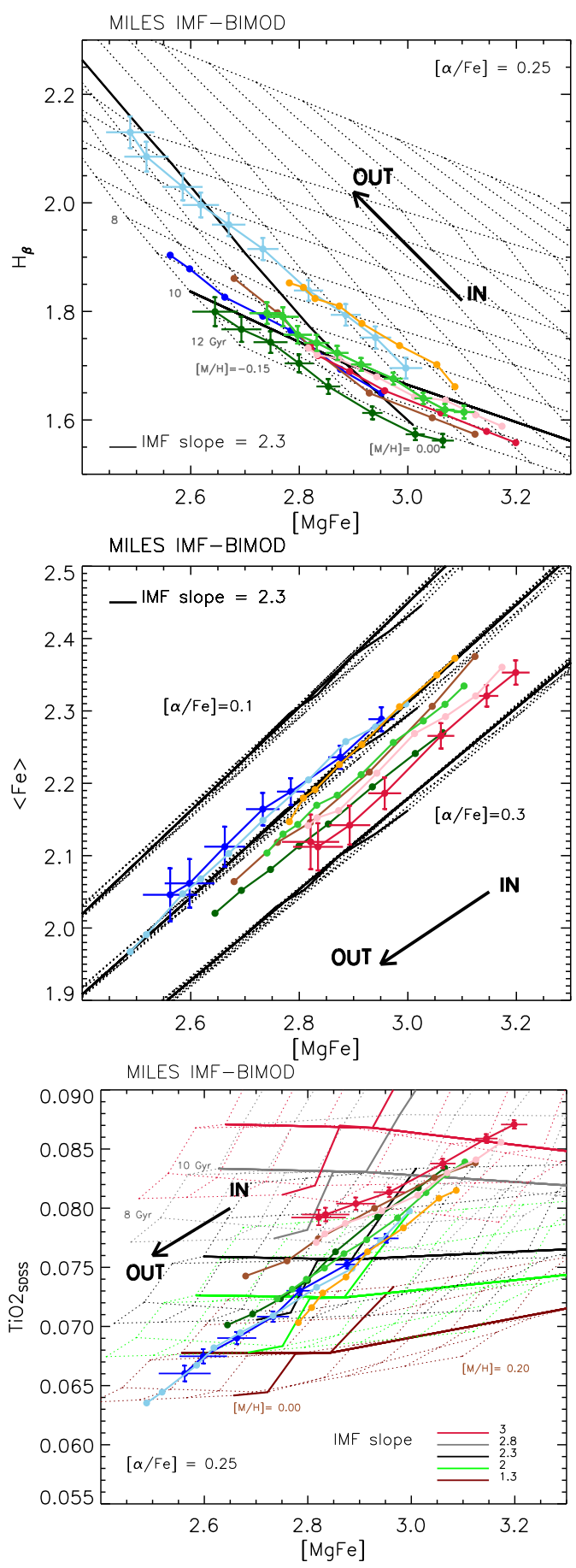

Figure 9. Lick indices $\mathrm{H}_{\beta},<\mathrm{Fe}>$ and $\mathrm{TiO} 2_{\mathrm{SDSS}}$ as a function of $[\mathrm{MgFe}]$ measured in the stacked spectra of the subsamples shown in Figure 2. Symbols of a fixed color show how index strength changes with distance from the center: $[\mathrm{MgFe}]$ decreases from center outwards (thick bold arrow indicates the direction of increasing galactocentric distance). Lighter shades (light blue, light green, orange and pink) represent galaxies with smaller sizes given their $\sigma_{0}$ and $L_{r}$; darker shades (dark blue, dark green, brown, red) represent galaxies with larger sizes. For a fixed IMF, the top panel is an age-metallicity diagnostic and the middle panel is an indicator of $\alpha$-enhancement. The bottom panel is used as an IMF diagnostic. In all three panels, SSP grids for some parameters fixed as labeled are shown. The smaller sizes, shown using lighter shades, clearly have stronger $\mathrm{H}_{\beta}$; they also have stronger $<\mathrm{Fe}>$ and slightly weaker $\mathrm{TiO}_{2}$ (except for B00). values reported in Figure 10 before subdividing the sample in SRs and FRs. In this format, Figure 10 highlights the fact that the smaller sizes have larger $\mathrm{H}_{\beta}$, and at fixed size, the FRs have larger $\mathrm{H}_{\beta}$. This difference between SRs and FRs is most pronounced for bin B11-S (orange symbols in bottom panel), and least pronounced for bin B10-L (dark green in middle panel). Similarly, Figure 11 shows that the larger galaxies in a bin are shifted down and to the right of the smaller galaxies (i.e. towards higher $[\alpha / \mathrm{Fe}]$ ), and SRs are shifted down and to the right of FRs of the same size.

It is interesting to compare these measurements for Es to those for the S0s that we had removed from bins B00 and B10. The filled and open inverted triangles in the top and middle panels show these S0s. S0s are FRs, and for bin B00-S the $\mathrm{H}_{\beta}-[\mathrm{MgFe}]$ values are similar to those of FR Es (light blue ellipses). While this suggests that the distinction between FR Es and S0s is not well motivated - consistent with Emsellem et al. (2011) and Cappellari (2016) - the $<\mathrm{Fe}>-[\mathrm{MgFe}]$ plot shows that the S0s tend to be slightly offset towards larger $\langle\mathrm{Fe}\rangle$. Also note that the central regions of S0s in bin B00-L (filled triangles) are more similar to the B00-L SR Es. Moreover, the differences between FR Es and S0s is larger in bin B10, which is why we removed S0s from all the analysis and interpretation which follows. It is clear that, had we included them, they would simply reinforce the differences we report between SR and FR Es.

\subsection{Stellar populations: Systematics}

As Paper I discusses, to interpret these measurements, we must make assumptions about how either the IMF or the different element enhancements $([\mathrm{X} / \mathrm{Fe}])$ depend on galaxy type. Specifically, the $\mathrm{TiO} 2_{\mathrm{SDSS}}-[\mathrm{MgFe}]$ model grids (the ones in the bottom panels of Figure 9) shift vertically as the value of $[\mathrm{X} / \mathrm{Fe}]$ varies (at fixed IMF). Since $[\mathrm{X} / \mathrm{Fe}]$ is not known a priori, this translates into uncertainty in the best-fit IMF. To proceed, we follow Paper I in making two rather different assumptions: one is that all galaxies have the same IMF, whatever their $\sigma_{0}$ and $L_{r}$, and that there are no IMF gradients within galaxies (following Paper I we refer to this as Assumption 1). In this case, the $\Delta_{[\mathrm{X} / \mathrm{Fe}]}$, i.e., the difference between the measured $\mathrm{TiO} 2_{\mathrm{SDSS}}$ from the stacked spectra and the value from the MILES model (with age, metallicity and $[\alpha / \mathrm{Fe}]$ given by e.g. Figure 9 ) due to variation in $[\mathrm{X} / \mathrm{Fe}]$ enhancements, shows strong gradients, which Paper I argues are unrealistic. The other is that $\Delta_{[\mathrm{X} / \mathrm{Fe}]}=0.003$ within a galaxy (Paper I refers to this as Assumption 3). In this case the IMF shows gradients.

One consequence of keeping $\Delta_{[\mathrm{X} / \mathrm{Fe}]}$ constant is that metal rich objects must have bottom-heavy IMFs (steeper slopes). In addition, there is a relatively strong degeneracy between the maximum age we allow and the IMF we infer. Our fiducial choice requires ages to be less than 13 Gyrs (maximum redshift of formation of $z=7$ ). However, if we set this upper limit to be 11 Gyrs (stars formed after redshift 2.5) then the inferred IMF is more bottom-heavy. In turn, this impacts the value of $M_{*} / L_{r}$ : more bottom heavy IMFs have larger $M_{*} / L_{r}$. Note that these shifts do not affect the rank ordering of the different bins. 

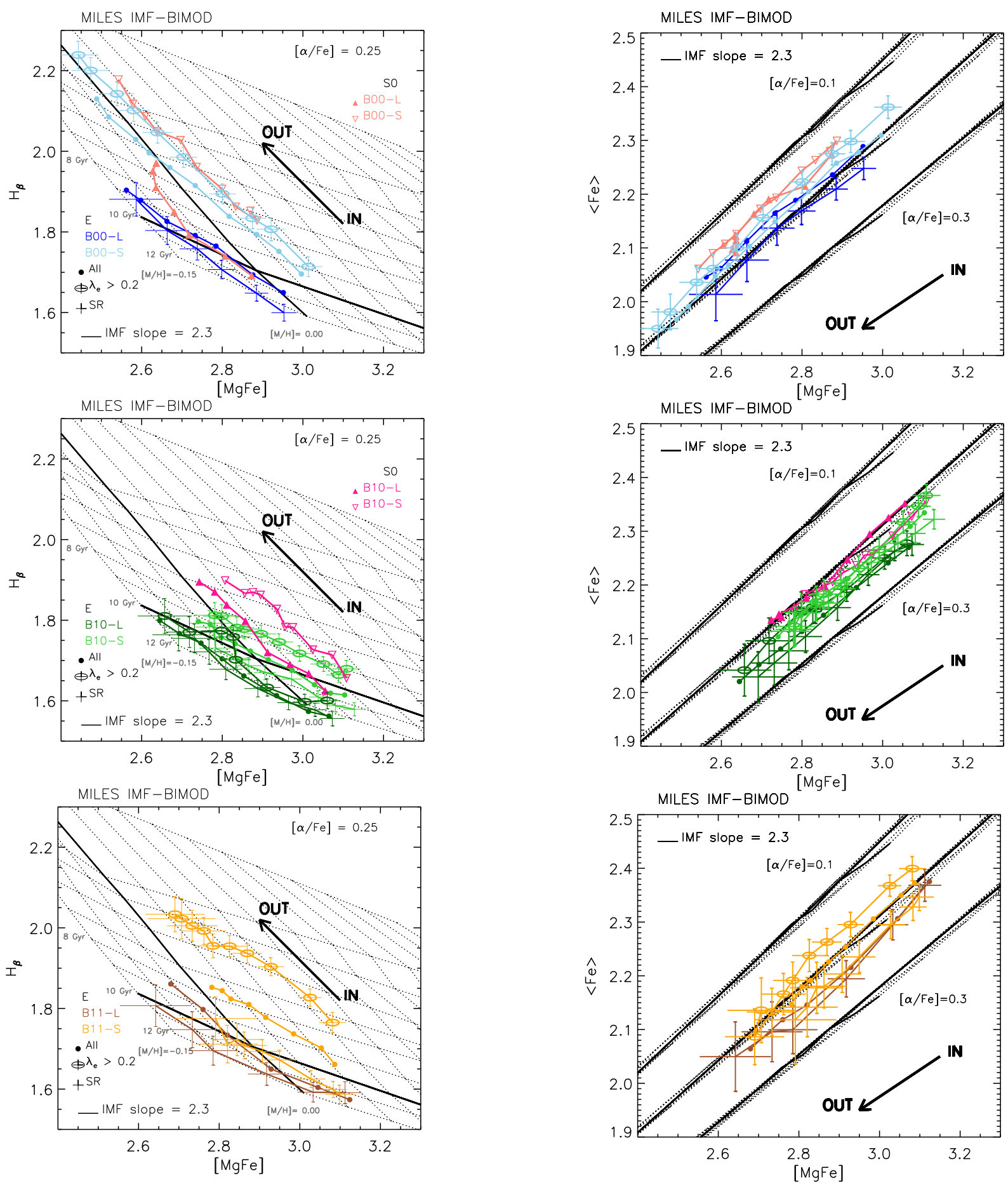

Figure 10. $\mathrm{H}_{\beta}$ vs $[\mathrm{MgFe}]$ for Es and S0s in bins B00 (top) and B10 (middle), and Es in B11 (bottom), subdivided by size (lighter shades show the smaller sizes in a bin) and further by rotation (crosses and ellipses show SRs and FRs). Fast rotators (ellipses) tend to have larger $\mathrm{H}_{\beta}$ for their $[\mathrm{MgFe}]$ than do slow rotators (crosses). SSP models interpret this as younger ages and larger metallicities for FRs. Filled circles (without error bars) show the values reported in Figure 10 before subdividing the sample in SRs and FRs. Filled and inverted open triangles in the top and middle panels show S0s which were classified as large or small using the same criteria as the Es.

Figure 11. Same as previous figure, but now showing $\langle\mathrm{Fe}\rangle$ vs $[\mathrm{MgFe}]$. In each bin, fast rotators (ellipses) tend to be less $\alpha$ enhanced.

\subsection{Stellar population trends with local velocity dispersion}

Figure 12 shows the SSP-inferred stellar population parameters in our four bins (left) and when subdivided by $R_{e}$ (right), for our second assumption about $\Delta_{[\mathrm{X} / \mathrm{Fe}]}$ (i.e, $\Delta_{[\mathrm{X} / \mathrm{Fe}]}=0.003$ within a galaxy). Symbols show the closestfitting IMF model (corresponding to the legend in the top left panel), but the actual value of IMF slope (as well as all 


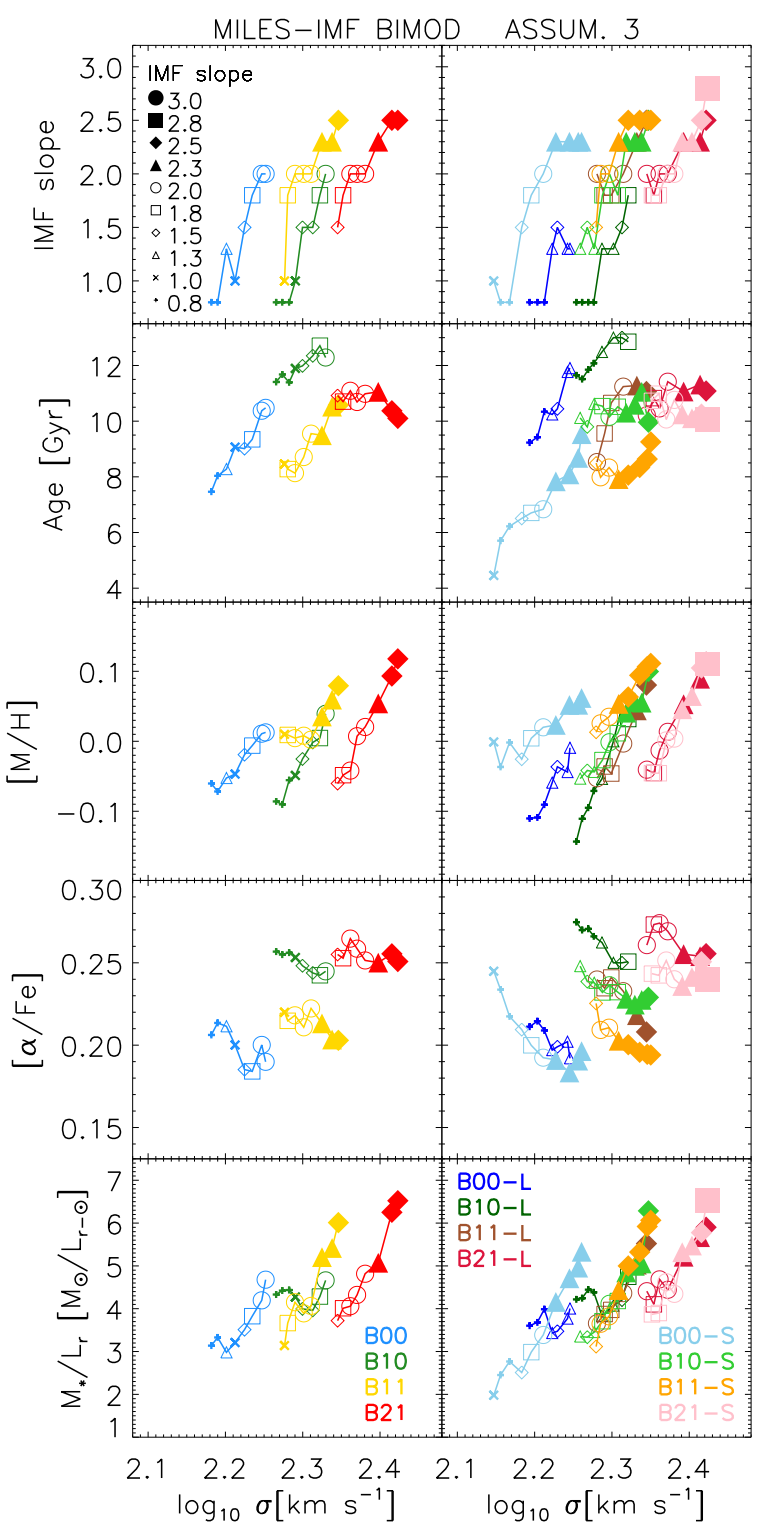

Figure 12. IMF slope, age, metallicity, $[\alpha / \mathrm{Fe}]$ and $M_{*} / L_{r}$ gradients in our four bins (left) and upon subdividing each by size (right), if we assume the IMF can differ from bin to bin, and vary within a galaxy, subject to the requirement that $\Delta_{[\mathrm{X} / \mathrm{Fe}]}=0.003$ in a galaxy. Different symbols indicate the slope (shown in the legend) of the closest IMF model, but all the properties (including the IMF slope) shown in the panels were inferred by interpolating between the two best-fitting IMF models.

the other inferred properties) shown is got by interpolating between the two best-fitting IMF models. The panels below them show the associated age, $[\mathrm{M} / \mathrm{H}],[\alpha / \mathrm{Fe}]$ and $M_{*} / L_{r}$ values which follow from the SSPs. These results are rather similar to those obtained by assuming the same IMF for all galaxies and no IMF gradients (i.e. our first assumption - we do not show a figure because we believe the required $\Delta_{[\mathrm{X} / \mathrm{Fe}]}$ gradients are unrealistic), except for $M_{*} / L_{r}$, which shows larger variations when IMF gradients are allowed (i.e., as in Figure 12).

The left hand panels reproduce results from Paper I. They show that:
- at fixed $L_{r}$, age increases with $\sigma$ (blue and green symbols trace out one curve, and yellow and red symbols another), but the higher $L_{r}$ objects tend to have smaller ages for the same $\sigma$ (green is older than yellow/red). However, we find an inverted age gradient for the most massive galaxies (bin B21): their centers are slightly younger;

- at fixed $L_{r},[\mathrm{M} / \mathrm{H}]$ does not depend on $\sigma$ (blue and green have same $[\mathrm{M} / \mathrm{H}]$, as do yellow and red), but the higher $L_{r}$ objects tend to have larger $[\mathrm{M} / \mathrm{H}]$ for the same $\sigma$ (blue and green have smaller $[\mathrm{M} / \mathrm{H}]$ than yellow and red);

- higher $\sigma$ galaxies have higher $[\alpha / \mathrm{Fe}]$ (red lies above green and yellow, which lie above blue), but at fixed $\sigma$, if $[\alpha / \mathrm{Fe}]$ is larger then so is age, but $[\mathrm{M} / \mathrm{H}]$ is smaller (compare yellow and green symbols in left hand panels: green is older and more enhanced, but less metal rich);

- $[\alpha / \mathrm{Fe}]$ does not increase, and in some cases decreases with increasing $\sigma$ within a galaxy.

- IMF slope and $[\mathrm{M} / \mathrm{H}]$ seem well correlated.

The trends with age, $[\alpha / \mathrm{Fe}]$ and $[\mathrm{M} / \mathrm{H}]$ are well-known (e.g. Bernardi et al. 2005, 2006; Graves et al. 2010; McDermid et al. 2015), and are usually taken to mean that higher $\sigma_{0}$ galaxies formed their stars on a shorter timescale (because $\log _{10}$ (timescale/Gyrs) $\approx 1.2-6[\alpha / \mathrm{Fe}]$, following, e.g., Thomas et al. 2005) and are less affected by supernovae (SN) feedback (because metallicity is low). The anti-correlation between $[\mathrm{M} / \mathrm{H}]$ and both age and $[\alpha / \mathrm{Fe}]$ at fixed $\sigma$ is the basis for arguing that $[\alpha / \mathrm{Fe}]$ is enhanced because Fe is suppressed, and not because $\alpha$ is enhanced. That IMF slope and $[\mathrm{M} / \mathrm{H}]$ are coupled is also in qualitative agreement with previous work: Martín-Navarro et al. (2015a) argue that IMF slope $\approx 2.2+3[\mathrm{M} / \mathrm{H}]$ for the BiModal models we are using here. We find this too, but do not show it because this correlation is model dependent, as we discuss in Paper I. The inversion of the age gradient for bin B21 is qualitatively consistent with Zibetti et al. (2019) which appeared while our paper was being refereed. However, as we describe shortly, this inversion is not obvious when we subdivide by size. Finally, as we noted in Paper I, it is surprising that the bin B21 galaxies (red), which have the largest $L_{r}$ and $\sigma_{0}$, are not the oldest. The oldest galaxies (green) are in bin B10; they also have low $[\mathrm{M} / \mathrm{H}]$ and anomalously high $[\alpha / \mathrm{Fe}]$ compared to the B11 (yellow) galaxies which have the same $\sigma_{0}$ but larger $L_{r}$

The right hand panels of Figure 12 show the result of splitting the bins shown in the left hand panels in two, based on size (Table 1). Galaxies with smaller than average sizes (for their $\sigma_{0}$ and $L_{r}$ ), which we show using lighter shades, are typically about 2 Gyrs younger (except perhaps for B21 which shows a smaller difference), slightly more metal rich (except perhaps for B21), and less $\alpha$-enhanced (except for B00), but with, nevertheless, little difference in $M_{*} / L_{r}$ (the age and metallicity effects approximately cancel). Note that the anti-correlation between either age or $[\alpha / \mathrm{Fe}]$ and $[\mathrm{M} / \mathrm{H}]$ at fixed $\sigma$ persists when split by size (except perhaps in bin B21). Bin B21, which includes galaxies with the largest $L_{r}$ and $\sigma_{0}$, shows the smallest differences. We will return to this in Section 3.4. However, note that the inversion of the age gradient for this bin, which is obvious in the left hand panel, is no longer present when we subdivide by size. The dependence on $R_{e}$ shows that there must be variations in star formation timescale and/or sensitivity to SN feedback even 


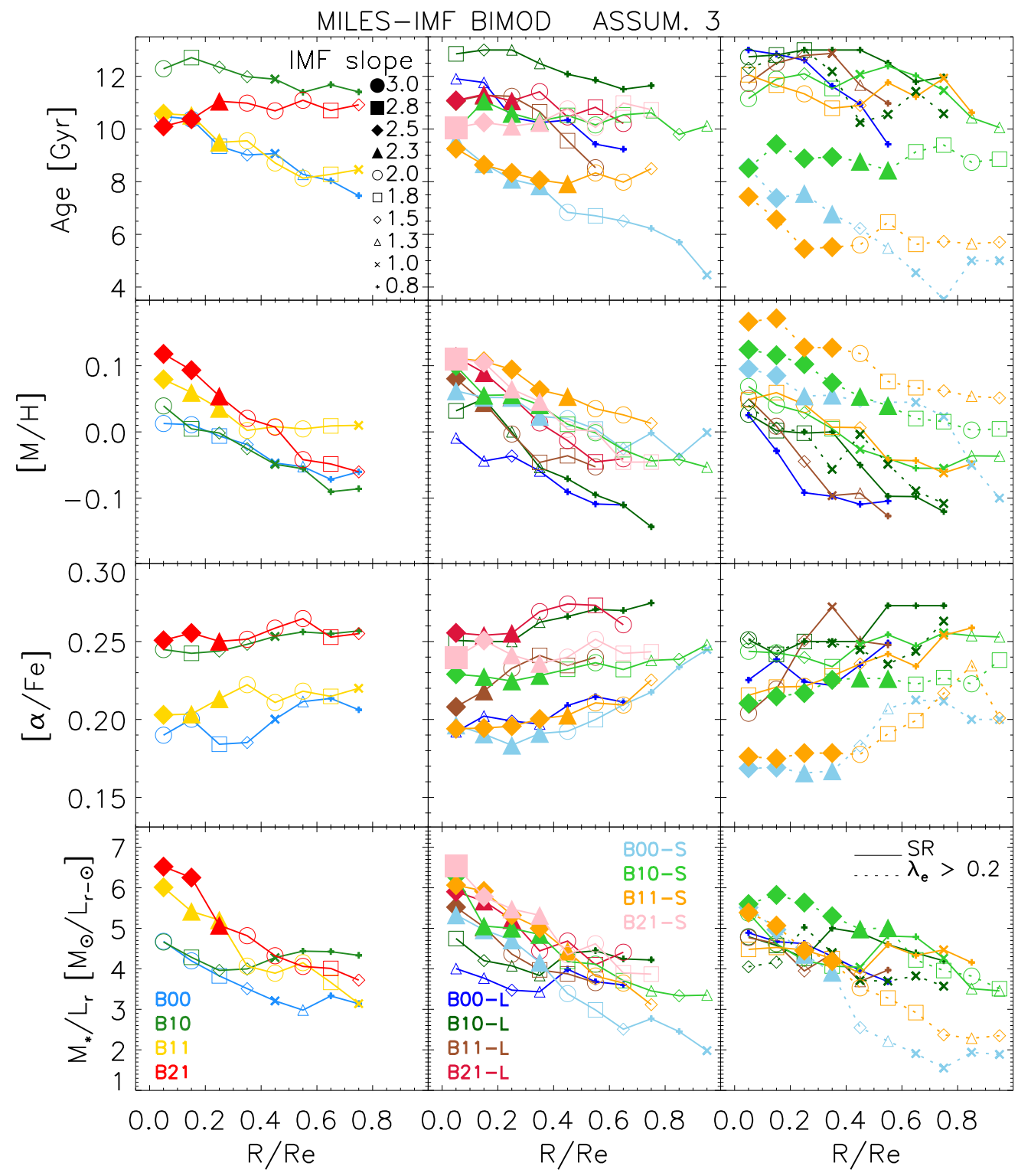

Figure 13. Age, metallicity, $\alpha$-enhancement and $M_{*} / L_{r}$ gradients when we allow IMF gradients (i.e. Assumption 3). Left and middle panels show the results of Figure 12 as a function of $R / R_{e}$ rather than $\sigma$. Color coding is same as before (lighter shades represent bins with smaller $R_{e}$ ), and symbols indicate different IMFs, as given in the legend. Right hand panel shows gradients in subsamples further divided on the basis of rotation; solid and dashed lines show SRs and FRs, respectively, in the bins for which we have sufficient statistics (see Table 1 and related discussion). To better highlight the difference between FRs and SRs we do not show the SRs of bins B21-L (red line) and B21-S (pink line) in the third column (although we do have sufficient statistics for the SRs of both bins) because they are dominated by SRs and so are almost indistinguishable from the B21-L and B21-S bins shown in the middle panels.

at fixed $\sigma_{0}$ : larger sizes had shorter timescales. The tests shown in Appendix A of Paper I indicate that these conclusions are robust against changes in the details of the SSP models, i.e. although there are differences in overall values, the differences regarding relative comparisons are small.

The differences between sizes (in a given $\sigma_{0}$ and $L_{r}$ bin) indicate that younger galaxies have higher surface brightnesses. While this is qualitatively consistent with previous work, in which surface-brightness was used explicitly (Graves et al. 2010, see Appendix B for more discussion), we believe that our binning in $\sigma_{0}$ and $L_{r}$ before subdividing in $R_{e}$ (rather than $\sigma_{0}$ and $R_{e}$ before subdividing in $I_{e}$ ) more effectively separates objects of similar masses. Moreover, as we discuss in the next subsection, we believe that rotation plays an important role.

It is clear that the oldest objects are those with larger than average sizes in B10-L, although the inner regions of B00-L objects are also surprisingly old: e.g. their age is similar (or even larger) than for B21 objects which have larger $L_{r}, \sigma_{0}$ and $R_{e}$. Note also that B10-S objects (light green) have approximately the same age, $[\mathrm{M} / \mathrm{H}]$ and $[\alpha / \mathrm{Fe}]$ as B11L objects (brown), even though they have different $L_{r}$ and very different sizes (c.f. Figure 2). We discuss these objects in Section 3.6. 


\subsection{Stellar populations of slow vs fast rotators}

We turn now to the gradients. The first two columns in Figure 13 show the same age, $[\mathrm{M} / \mathrm{H}],[\alpha / \mathrm{Fe}]$ and $M_{*} / L_{r}$ values as Figure 12, but now as a function of $R$ rather than $\sigma(R)$.

The third column of Figure 13 shows a similar analysis of the slow and fast rotators (solid and dashed lines) in all bins where we have sufficient statistics. Whereas we do have sufficient statistics for the B21 SRs (see Section 2.2), we do not show them in the third column because their populations are almost indistinguishable from the B21-L and B21-S bins shown in the middle panels (this better highlights the difference between FRs and SRs for the other bins).

Before we discuss the FRs and SRs in the right hand column, notice that plotting versus $R$ makes it obvious that:

- In all bins, the larger galaxies are older, more metal poor and more $\alpha$-enhanced (middle panels);

- In all bins, age and metallicity increase towards the central regions, with metallicity gradients generally being stronger; age gradients are strongest in B00 and weak otherwise;

- $[\alpha / \mathrm{Fe}]$ gradients are weak, with the centers being less enhanced;

- $M_{*} / L_{r}$ gradients are stronger in bins B11 and B21, but weak or non-existent in the other two bins (bottom left), in agreement with expectations from a very different analysis (Bernardi et al. 2018b); when subdivided by size (bottom middle) gradients are present in all four of the smaller bins and in bins B11-L and B21-L, but are non-existent in bins B00-L and B10-L;

- B10 galaxies are the oldest and most metal poor (left hand columns), with B10-L galaxies being the oldest but not quite the most metal poor (middle columns);

- The most metal poor objects are in B00-L, and the central regions of these galaxies are remarkably old (middle panels).

In the right hand column, many of these trends are even more dramatic:

- SRs (solid) are significantly older (as much as $\sim 5$ Gyrs), less metal rich and more $\alpha$-enhanced than FRs in all our bins except for the B10-L FRs (dark green dashed line) which behaves more similarly to the SRs;

- The central regions of SRs have almost the same age and metallicity in all our bins except for B21 (compare red and pink lines in middle panels with solid lines in the right hand panels); the B21 SRs are the SRs with the youngest ages and largest metallicities even though they have the largest $L_{r}$ and $\sigma_{0}$;

- Except for bin B00, the other SR bins show rather flat age gradients but stronger $[\mathrm{M} / \mathrm{H}]$ gradients;

- FRs show slighlty stronger $[\alpha / \mathrm{Fe}]$ gradients than SRs, with the centers being less enhanced;

- FRs show slightly stronger $M_{*} / L_{r}$ gradients than SRs. Only SRs with large $L_{r}$ and $\sigma_{0}$ (i.e. B21-S in middle panel) show comparable $M_{*} / L_{r}$ gradients to FRs.

- The stellar populations in all the B10 bins are remarkably similar, with only the B10-S FRs having smaller ages and higher metallicities; as a result, compared to the other FRs, those in bin B10-L are anomalously old, metal poor and $\alpha$ enhanced.

As the SRs in a bin tend to be larger than FRs (c.f. Figure 7), it appears that many of the differences between large

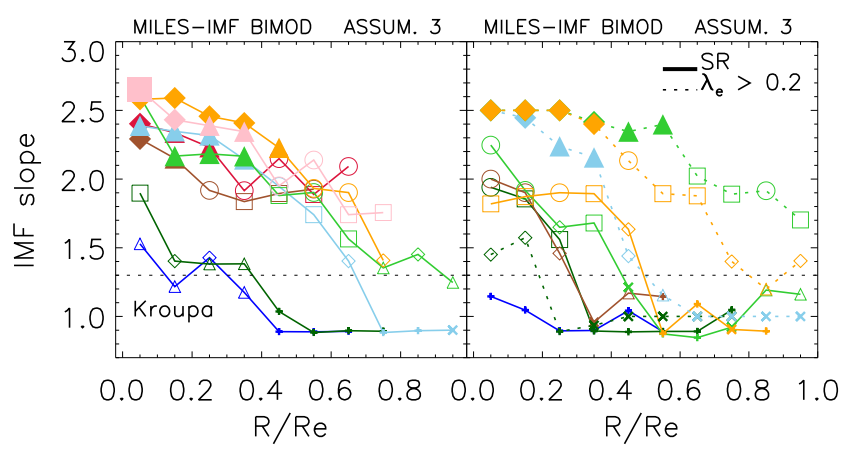

Figure 14. Inferred variation in IMF slope for samples split by size (left) and rotation (right). Symbols (same as top left panel of Figure 13) show the closest IMF model, but the IMF slope shown is got by interpolating between the two closest IMF models. Objects with smaller than average sizes for their $L_{r}$ and $\sigma_{0}$ (lighter shades) tend to have steeper (more bottom heavy) IMFs; bins B00-L and B10-L (dark blue and green) have the smallest IMF slopes. With the exception of the B10-L bin (dark green) FRs (dashed) tend to have steeper (more bottom heavy) IMFs than SRs.

and small sizes in the middle panels of Figure 13 are more strongly correlated with differences in rotation. In particular, SRs formed their stars rapidly, at approximately the same time, long ago, and so have sub-solar metallicities (except for bin B21). In contrast, FRs are significantly (as much as $\sim 5$ Gyrs) younger, formed their stars over a longer timescale, and so are more metal rich (although recall that these are light-weighted conclusions that are dominated by the younger stars). In this respect, our FR ages are rather different from those reported by the ATLAS ${ }^{3 \mathrm{D}}$ collaboration. Figure 11 of McDermid et al. (2015) shows that they estimate the vast majority of FRs with $\sigma \geq 220 \mathrm{kms}^{-1}$ to be more than 10 Gyrs old. Few of our FRs are this old. We return to this in Section 3.7.

We remarked that it is curious that the B21 SRs are younger and more metal rich than the other SRs. In view of our comments about SSP systematics in Section 3.2, it should come as no surprise that this bin has a different IMF than other SRs. Figure 14 shows the gradient in the IMF slope parameter associated with the SSP parameters shown in the middle and right hand panels of Figure 13. (The symbols shows the closest-fitting IMF model, but the actual slope shown is got from interpolating between the two best-fitting IMF models.) The slope is larger - indicating a more bottom-heavy IMF - in the central regions of all the bins. In addition, the right panel shows that FRs (dashed lines) tend to have steeper slopes than SRs (solid). However, the IMFs for B21 (red and pink in the left panel), which are dominated by SRs, are more like those of FRs. In contrast, the objects in bins B00-L and B10-L - the two oldest bins with the lowest metallicities in the middle panels of Figure 13 - have the shallowest IMFs. These results suggest that metallicity plays a major role in setting the IMF (also see Figure 17 in Paper I). 


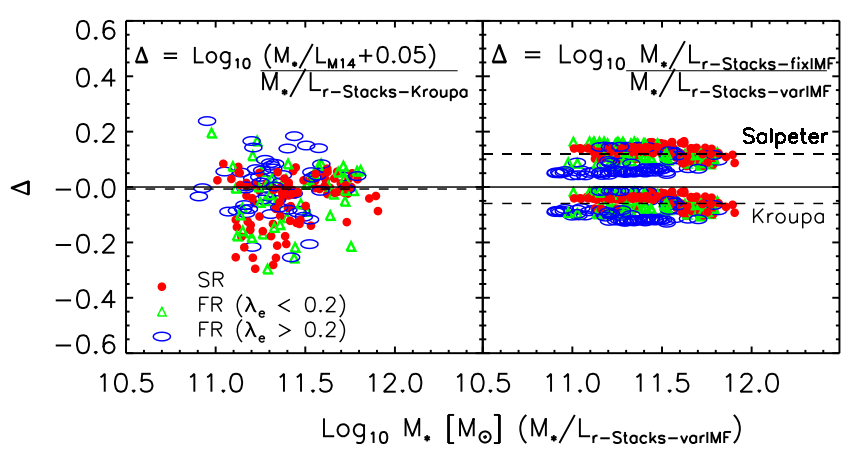

Figure 15. Comparison of integrated $M_{*} / L_{r}$ estimates. Red circles and blue ellipses show SRs and FRs with $\lambda_{e}>0.2$; green triangles show the other FRs; dashed lines show the median trends. Left: Ratio of integrated $M_{*} / L_{r}$ from Mendel et al. (2014) (shifted by 0.05 dex to transform from a Chabrier to a Kroupa IMF) to our own estimate in which we assume the IMF is Kroupa on all scales for all objects. This ratio is shown as a function of the mass estimate which combines each galaxies $L_{r}$ with the $M_{*} / L_{r}$ profile that is appropriate for its $L, \sigma_{0}, R_{e}$ and $\lambda_{e}$ (shown in Figure 13). Right: Ratio of integrated $M_{*} / L_{r}$ estimates when we assume all galaxies have the same IMF (Kroupa or Salpeter) on all scales, to that when the IMF is allowed to vary within a galaxy and across the population.

\subsection{Stellar and dynamical masses of slow and fast rotators}

With $M_{*} / L_{r}$ gradients in hand, we can attempt a selfconsistent comparison of stellar and dynamical masses. This is interesting because, when these gradients are ignored, then stellar population estimates of $M_{*}$ which assume a Kroupa or Chabrier IMF tend to lie about 0.2 dex below $M_{\text {dyn }}$ estimated from a Jeans equation analysis. Some have attributed this discrepancy to the IMF, rather than to problems with the $M_{\mathrm{dyn}}$ estimate (see Paper I for a more detailed discussion).

Our first step is to compare our integrated $M_{*} / L_{r}$ estimates (which are computed by dividing the total $M_{*}$ by the total luminosity $L_{r}$, see equation 3 in Paper I and related discussion) with those from the literature. (Comparing integrated $M_{*} / L_{r}$ rather than $M_{*}$ estimates themselves removes systematics associated with the total luminosity $L_{r}$, see Bernardi et al. 2013, 2017a,b; Fischer et al. 2017.) The left hand panel of Figure 15 shows the ratio of the integrated $M_{*} / L_{r}$ estimate from Mendel et al. (2014) (shifted by 0.05 dex to transform from their Chabrier IMF to a Kroupa IMF) to that returned by our analysis if we assume the IMF is Kroupa for all galaxies, and there are no IMF gradients (there may still be $M_{*} / L_{r}$ gradients). We show this ratio as a function of our $M_{*}$ estimate in which we include the full $M_{*} / L_{r}$ gradient (i.e. when IMF variations are allowed). Red circles show SRs, blue ellipses show FRs with $\lambda_{e}>0.2$ and green triangles show the remaining objects (i.e. FRs with small $\lambda_{e}$ ). The agreement is rather good, thus establishing consistency with the literature.

The right hand panel shows the ratio of the integrated $M_{*} / L_{r}$ estimates when we fix the IMF to Kroupa or Salpeter to that when we allow the IMF to vary (both within a galaxy and between bins). This shows that fixing the IMF to Kroupa underestimates $M_{*}$ by about 0.05 dex, whereas fixing it to Salpeter overestimates it by about 0.15 dex. In addition, the fact that the red points lie slightly above the blue mainly reflects the fact that SRs have slightly larger $M_{*} / L$ at large $R$ (bottom right panel of Figure 13). Note that even when the IMF in the central regions is quite different (Figure 14), the net effect on the integrated $M_{*} / L_{r}$ is small.

We now compare our (variable IMF) $M_{*}$ values with three different estimates of the dynamical mass, and present the results as $M_{\mathrm{dyn}} / M_{*}$ vs $\sigma_{e}$, where $\sigma_{e}$ is the velocity dispersion within $R_{e}$ (as described in Paper I, here we show galaxies with FLAG_FIT $=1$, i.e. $\sim 64 \%$ of our E sample). The left hand panel of Figure 16 sets $M_{\mathrm{dyn}}=4 R_{e} \sigma_{0}^{2} / G \approx 5 R_{e} \sigma_{e}^{2} / G$ (e.g. McDermid et al. 2015). On average, $M_{\text {dyn }}$ lies about 0.2 dex above our $M_{*}$ estimates although the ratio $M_{\mathrm{dyn}} / M_{*}$ tends to increase with $\sigma_{e}$. The dotted lines show linear fits to this correlation and the dot-dashed lines show the correlation reported by Li et al. (2017) (here we have shifted their relation by 0.13 dex to account for the fact that the Salpeter IMF, their fiducial choice, has $\Delta \sim 0.13$ in the right hand panel of our Figure 15). This correlation has been used to argue that the IMF of massive galaxies becomes more bottomheavy (Salpeter-like) at large $\sigma_{e}$ (e.g. Cappellari et al. 2013; $\mathrm{Li}$ et al. 2017). However, our analysis shows that the IMF is only bottom heavy in the central regions.

The middle panel shows $k(n, R) R_{e} \sigma_{0}^{2} / G$, where $k$ is taken from Table 1 of Bernardi et al. (2018a). This estimate accounts for the fact that the light profile shape (parameterized by the Sersic index $n$ ) differs from one galaxy to another, but assumes that $M_{*} / L$ is a constant that is fixed by asking that the Jeans equation estimate of the projected velocity dispersion within $R_{e} / 10$ match the observed $\sigma_{0}$. In this case the $M_{\mathrm{dyn}} / M_{*}-\sigma_{e}$ correlation is in even better agreement with Li et al. (2017): if anything, the correlation is slightly tighter.

The right hand panel of Figure 16 shows the result of including the $M_{*} / L$ profile in our Jeans equation analysis (see Paper I for details). There are three remarkable differences with respect to the other two panels. First, the mean offset is gone: accounting for $M_{*} / L_{r}$ gradients reduces $M_{\mathrm{dyn}}$ by about 0.2 dex, bringing it into good agreement with $M_{*}$ estimated self-consistently using the same gradients. Second, the slope of the $M_{\mathrm{dyn}} / M_{*}-\sigma_{e}$ relation is significantly flatter for SRs (red dotted) and steeper for the fastest FRs (blue dotted). Third, the scatter is smallest for the SRs (red circles) and largest for the fastest FRs (blue ellipses). These last two points may be a consequence of the fact that our Jeans equation analysis assumes no rotation, and this is a worse approximation when $\lambda_{e}$ is large. In addition, it may be that single SSPs are worse approximations to the star formation histories of FRs than they are for SRs.

To summarize: Self-consistently accounting for gradients when estimating $M_{*}$ and $M_{\mathrm{dyn}}$ yields good agreement between the two, confirming what we found in Paper I. In particular, this agreement comes because gradients reduce the $M_{\text {dyn }}$ estimate (more than they increase $M_{*}$ ). As we noted in Paper I, this agreement implies that we can now specify the stellar mass scale identified by Bernardi et al. (2011), $2 \times 10^{11} M_{\odot}$ if the IMF were Chabrier, without also specifying an IMF. The offset of $\sim 0.05$ dex from Chabrier to Kroupa combined with the $\sim 0.05$ dex offset between 


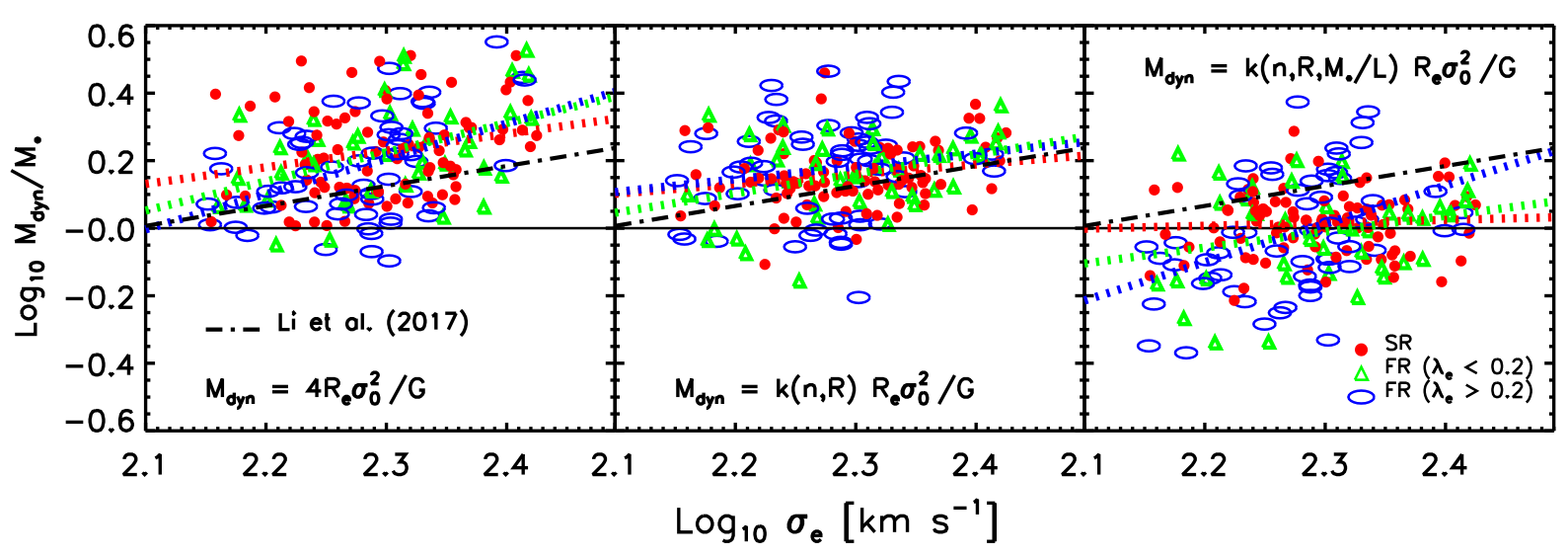

Figure 16. Comparison of three dynamical mass estimates with our variable IMF stellar mass estimate, in which IMF gradients contribute to $M_{*} / L_{r}$ gradients, shown versus $\sigma_{e}$. All three panels have $M_{\mathrm{dyn}} \propto R_{e} \sigma_{0}^{2} / G$ with the proportionality factor: (left) constant, the same for all galaxies; (middle) dependent on the light profile shape, but ignoring $M_{*} / L_{r}$ gradients; (right) dependent on the product of the light profile and the same $M_{*} / L_{r}$ profile which was used to estimate $M_{*}$. Red circles and blue ellipses show SRs and FRs with $\lambda_{e}>0.2$; green triangles show the other FRs; dotted colored lines show how the ratio $M_{\mathrm{dyn}} / M_{*}$ scales with $\sigma_{e}$ for each subsample; dot-dashed line shows the correlation reported by Li et al. (2017), offset slightly to account for the fact that the Salpeter IMF (their fiducial choice) has $\Delta \sim 0.13$ in the right hand panel of our Figure 15. In the right hand panel the offset is gone: self-consistently accounting for $M_{*} / L_{r}$ gradients brings $M_{\mathrm{dyn}}$ into good agreement with $M_{*}$. In addition, the red dotted line (SRs) is significantly flatter than the blue dotted line (FRs), a consequence of the fact that our Jeans equation analysis assumes no rotation, and this is a worse approximation when $\lambda_{e}$ is large.

Kroupa and our variable IMF estimate (right hand panel of Figure 15), suggest that this scale is more like $3 \times 10^{11} M_{\odot}$.

\subsection{The galaxies in bin B10}

For completeness, we now discuss the objects in bin B10, which were identified in Paper I as being anomalously old (top left panel of Figure 13). The top middle panel of Figure 13 suggests that, in fact, it is the larger B10-L objects which are extreme - the smaller B10-S objects are not particularly unusual (although they are unusually old compared to the smaller objects in the other bins). Morever, the top right panel of Figure 13 shows that it is rotation, not size, which seems to matter. This is because the B10 SRs are not particularly different from the SRs in the other bins. In this respect, the question is no longer: 'Why are the B10 Es so old?' but 'Why are the B10-L FRs so old?' and 'Why are all the sub-bins of B10 older?' While we do not have good answers to these questions, we can at least address the question of why there has been no previous discussion of anomalously old galaxies with with $\sigma_{0} \leq 250 \mathrm{~km} \mathrm{~s}^{-1}$ and $-22.5 \geq M_{r} \geq-23.5$ (stellar masses of $\left.1 \times 10^{11} \leq M_{*} / M_{\odot} \leq 3 \times 10^{11}\right)$.

One of the key differences between previous work with 'early-types' and ours with Es is that we have removed S0s. While there were essentially no S0s in bins B11 and B21 (Figure 1), they were present in, and removed from, our lower $L_{r}$ bins (Figures 2 and 4 ). We have already shown some differences between S0s and Es (e.g. Figures 5 and 6). In addition, Figures 10 and 11 show that, compared to Es in bin B10, the S0s have stronger $\mathrm{H}_{\beta}$ and $<\mathrm{Fe}>$ at a give $[\mathrm{MgFe}]$. Single stellar population model fits to S0s would return younger ages, higher metallicities, and smaller $\alpha$-enhancements than for Es. Hence, adding the S0s to our sample of Es would reduce the anomalously large ages for bin B10 and, to some extent, the SRs in bin B00-L as well.
As there are very few S0s in bins B11 and B21, the question of what happens if they are included is moot. We conclude that it would have been more difficult to notice that bin B10 is anomalous if we had not removed its S0s.

\subsection{Ages, sizes and the SR/FR dichotomy}

The SR/FR dichotomy has important implications for galaxy scaling relations. To illustrate, Figure 17 shows the correlation between size and stellar mass, with colors indicating age. (The age of each galaxy is obtained by weighting the age profile shown in the top right panel of Figure 13 that is appropriate for its $L_{r}$ and $\sigma_{0}$ with its surface brightness profile.) The three panels show FRs with $\lambda_{e}>0.2$ (left), FRs with $\lambda_{e}<0.2$ (middle) and SRs (right). Comparison of the left and right panels shows clearly that FRs are younger than SRs of the same mass, as might have been expected from the dichotomy in the top right panel of Figure 13.

The vertical dotted line in Figure 17 shows the mass scale identified by Bernardi et al. (2011), which we noted is $3 \times 10^{11} M_{\odot}$ after accounting for the fact that the IMF is variable. Above this mass, most SRs and FRs lie above the solid line shown, indicating that the $R_{e}-M_{*}$ relation steepens. In addition, there are few FRs above this mass. That these mass scales coincide is consistent with ATLAS ${ }^{3 \mathrm{D}}$ (Cappellari et al. 2013), although the value of this mass scale differs from what they report, since our $M_{*}$ estimates are different (e.g. Figure 16).

It is interesting to contrast this with Figure 18, in the right hand panel of which we have simply stacked together all three bottom panels of Figure 17. While this shows that a wide variety of ages contributes to each $R_{e}$ and $M_{*}$ bin, it appears that if one averages the SRs and FRs together, then one would still find that the smaller objects are younger (mainly because smaller galaxies tend to be FRs). Now consider the left hand panel. In this case, we have colored ob- 


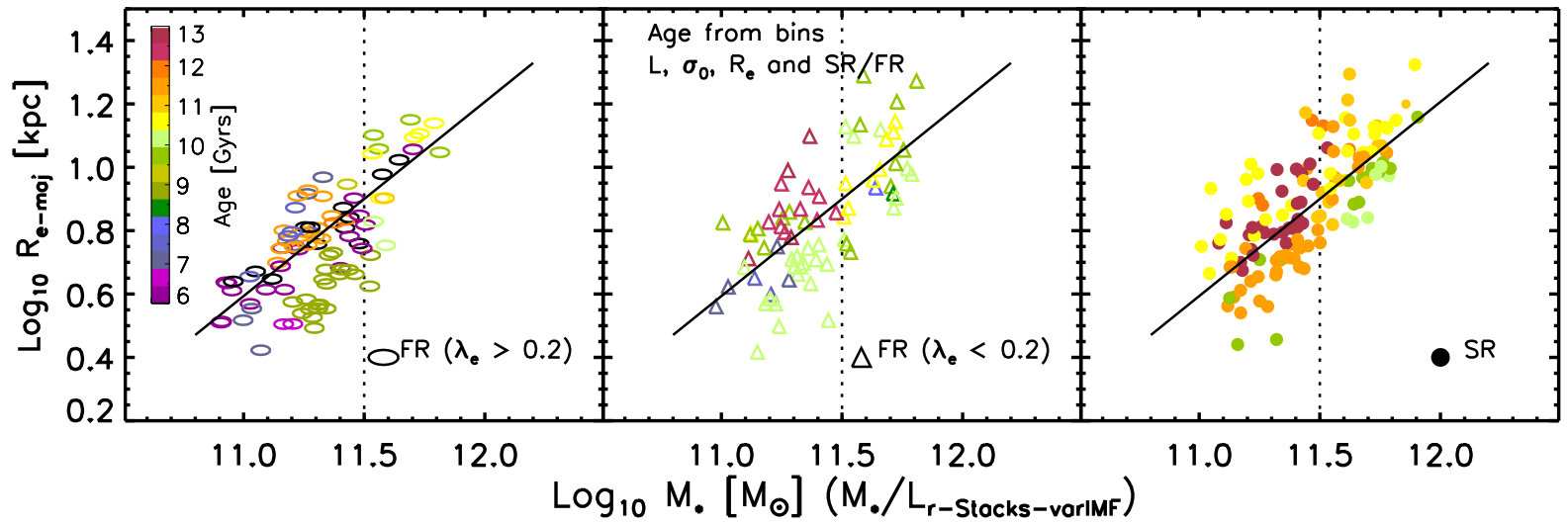

Figure 17. Size-stellar mass correlation for FRs with $\lambda_{e}>0.2$ (left), FRs with $\lambda_{e}<0.2$ (middle) and SRs (right), with symbol color indicating light-weighted age. FRs are younger than SRs of the same mass. Solid line shows the best linear fit of the size-mass relation to the full sample. Dotted vertical line shows the mass scale identified by Bernardi et al. 2011 (after accounting for the fact that the $\mathrm{IMF}$ is variable). Above this mass, the number of objects below the solid line drops, indicating that the slope of the $R_{e}-M_{*}$ correlation steepens. In addition, above this mass the SR/FR ratio increases.

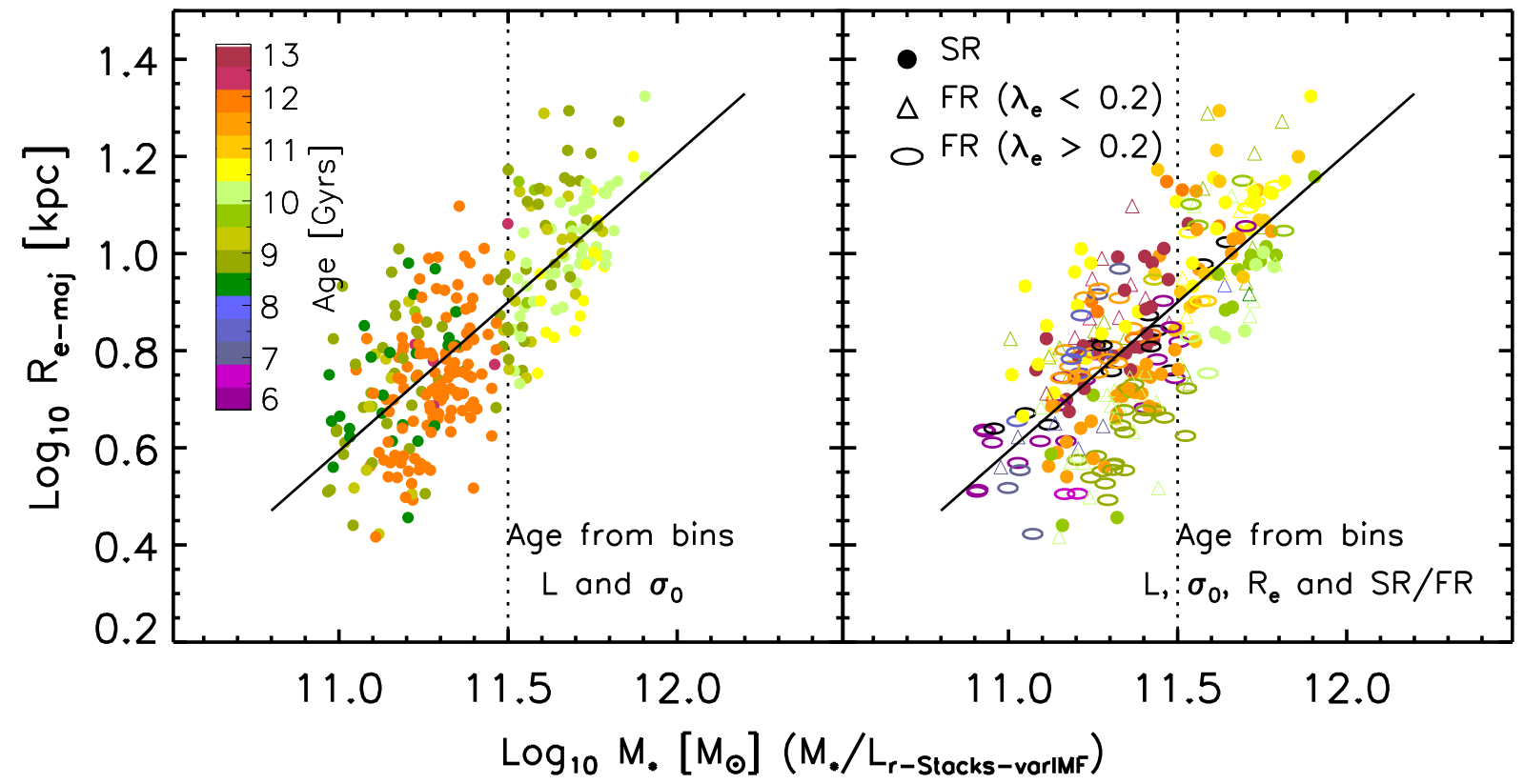

Figure 18. Correlation between size and stellar mass with symbols colored by light-weighted age, where age in left and right panels is estimated from the profiles shown in the top left and top right panels of Figure 13, respectively. (Note that right panel simply shows all the symbols from all the panels of Figure 17.) Left panel suggests that more compact galaxies are older; right panel suggests almost the opposite.

jects by the ages estimated from the four $\left(L, \sigma_{0}\right)$ stacks without subdividing by size or rotation. (I.e., in this case the age estimates use the profiles in the top left rather than top right panel of Figure 13.) This shows that, at fixed $M_{*}$, smaller galaxies are older, not younger. Evidently, how one does the averaging matters. The age from the average stack (left) is not the same as averaging the ages from many stacks (right). This difference should be borne in mind in future analyses.

Before ending this subsection, it is worth noting that smaller galaxies being older is consistent with one of the major results of Paper XXX of the ATLAS ${ }^{3 \mathrm{D}}$ collaboration (e.g. Figure 6 of McDermid et al. 2015, but note that our sample only probes the largest masses and velocity dispersions). While the left hand panel of our Figure 18 appears to be consistent with this, their analysis, based on averaging the ages of individual galaxies, should be closer to the trend observed in the right hand panel, which indicates that compact galaxies are younger, not older. For us, especially at smaller $M_{*}$, this is driven by the fact that compact galaxies tend to be FRs, and FRs are younger. As we noted earlier, McDermid et al. (2015) report much older FRs. This difference in the ages of FRs is the main reason our conclusion about the size-mass-age correlation differs from theirs. We provide more discussion of how our results compare with those of ATLAS ${ }^{3 \mathrm{D}}$ in Appendix B. 

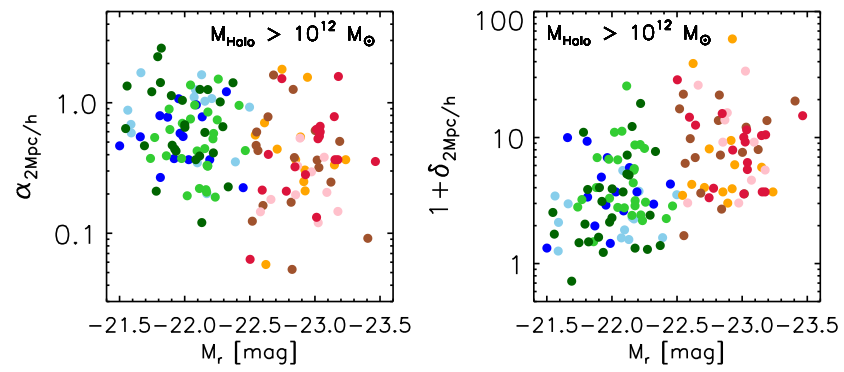

Figure 19. Fainter galaxies in our sample are in lower density regions (right) with stronger tidal fields (left).

\section{DOES ENVIRONMENT MATTER?}

We now check if the SR/FR dichotomy is correlated with environment.

\subsection{Environment: Large-scale density and tidal fields}

In this subsection, we use two measures of the environment, both provided by Paranjape et al. (2018): one is a measure of the density smoothed over a few $\mathrm{Mpc}$, and the other is a measure of the tidal field strength smoothed over the same scale. (These measures are only available for approximately half of our sample.) Figure 19 shows that, as expected, the lower $L_{r}$ bins are in less dense environments where the tidal field is slightly stronger. However, we find no striking difference between the environments of the two low- $L_{r}$ bins (B00 and B10).

\subsection{Environment: Central vs satellite}

Figures 20 and 21 show the result of a different test. Here, we have matched our galaxies to the group catalog of Yang et al. (2007, hereafter Yang+), and classified galaxies by whether they are central galaxies in their group or not. Thus, here, the 'environment' is defined on a smaller scale than before.

In the groups which Yang+ estimate to have halo masses in excess of $10^{13} M_{\odot}$, there are 1550 central Es with $-22.5 \leq M_{r} \leq-23.5$ and only 450 satellite Es (morphological classification for the SDSS galaxies in the Yang+ sample comes from Domínguez Sánchez et al. 2018). In this luminosity range, most Es are centrals; one in three or four is a satellite. However, for $-21.5 \leq M_{r} \leq-22.5$, there are 500 central Es but 1750 satellites: there are more than three times as many satellite Es as centrals. Evidently, at $M_{r} \approx-22.5$ $\left(M_{*} \sim 3 \times 10^{11} M_{\odot}\right)$, there is a significant change in the $\mathrm{E}$ population: brighter than this, most Es are centrals; fainter than this, they tend to be satellites. Mergers are expected to have played a role in the assembly and star formation histories of central galaxies in clusters, so it is probably no coincidence that this is the luminosity scale at which earlytype galaxy scaling relations change (Bernardi et al. 2011). Since bins B10 and B11 are separated by this critical luminosity $\left(M_{r} \sim-22.5\right)$ but have same $\sigma_{0}$, it is interesting to check how their group environments compare.

Before we do so, there is an interesting fact about MaNGA Es which has not been highlighted before: In haloes more massive than $10^{13} M_{\odot}$, MaNGA has 15 centrals and

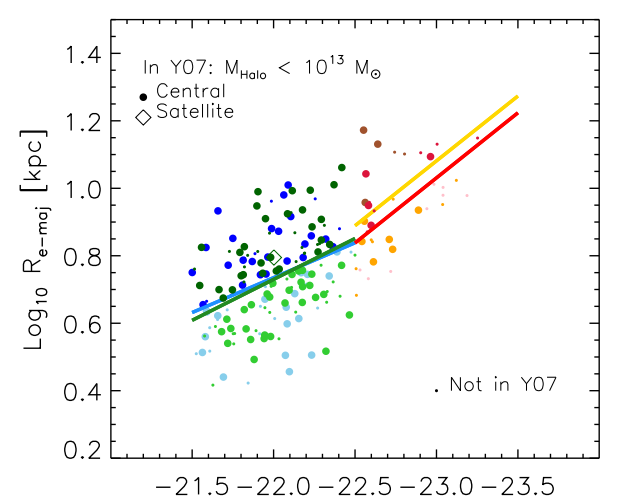

$M_{r}[\mathrm{mag}]$

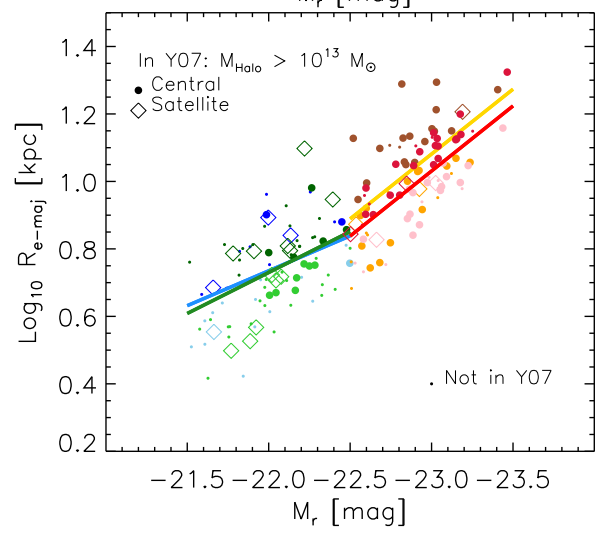

Figure 20. Size-luminosity relation of the MaNGA E galaxies in groups identified by Yang + as being less (top) or more (bottom) massive than $10^{13} M_{\odot}$. Filled circles and open diamonds show MaNGA Es identified as central and satellite galaxies in the groups; small dots show MaNGA Es that are not in the group catalog.
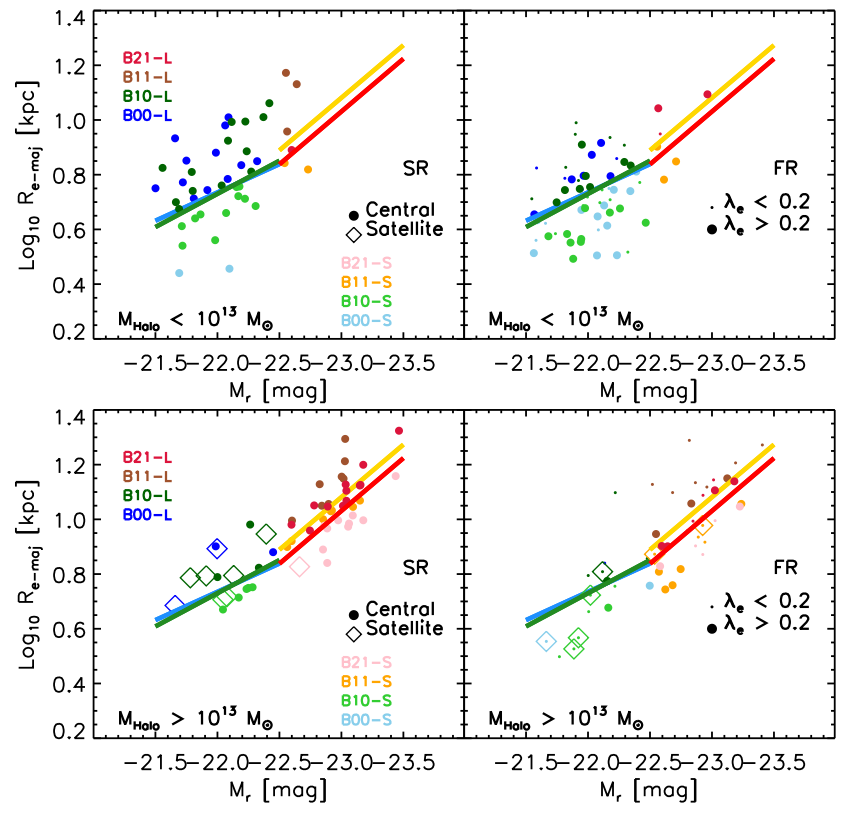

Figure 21. Same as previous figure, but now subdivided into SRs (left) and FRs (right). 
16 satellites for $M_{r}$ between -21.5 and -22.5 and 64 centrals and 7 satellites for $M_{r}$ between -22.5 and -23.5 (205 centrals and 14 satellites if we do not limit the sample to $z<0.08)$. Thus, satellite Es are very under-represented in MaNGA compared to in the full Yang catalog. Therefore, it is a good approximation to think of MaNGA Es as being dominated by centrals in all bins.

Figure 20 shows that low luminosity Es (B00 and B10) tend to be centrals of low mass halos (top panel), whereas high luminosity Es (B11 and B21) are centrals of more massive halos (bottom panel). B00 and B10 objects in massive halos tend to be satellites. These are not surprising trends, since group mass and central galaxy luminosity are known to be well-correlated. (A number of MaNGA galaxies are not in the group catalog: we have shown them as small dots in both panels.)

The process of becoming a central galaxy is generally believed to involve mergers (e.g. Mo et al. 2010), though whether these are major or minor and if they are between similar morphological types or not is still debated (e.g. Bundy et al. 2009; Hilz et al. 2013; Behroozi et al. 2015; Man et al. 2016). Presumably this has affected the Es in bins B11 and B21. In contrast, the B00 and B10 Es are primarily centrals of lower mass halos. These halos are more isolated, suggesting they have not undergone significant recent merger activity. With this in mind, it is interesting to ask if there is any correlation between SR or FRs and halo mass. Figure 21 shows the same $R_{e}-L_{r}$ correlation as before, but now for SRs (left) and FRs (right) in low (top) and high (bottom) mass groups. Unfortunately, the numbers are too small to allow clear conclusions. For example, while the ratio of FRs to SRs in each bin is slightly reduced in the more massive groups, the sample is too small to draw firm conclusions, especially given that MaNGA tends to pick centrals, and not all MaNGA objects are present in the group catalog.

Nevertheless, our results are consistent with the statements that both the SRs and the FRs in B10 are older and less metal rich than objects in B11 because they formed in lower mass halos. In addition, our results showing that the FRs in bin B11 appear younger and more metal rich than those in bin B10 support a senario in which these FRs may be the result from a relatively recent major merger between a fast rotator (S or $\mathrm{S} 0$ ) and an $\mathrm{E}$.

\section{DISCUSSION}

Appendix B discusses how our findings compare with previous work. This section provides a brief summary. We describe global quantities before describing gradients.

We find that at fixed $L_{r}$ and $\sigma_{0}$, the objects with larger $R_{e}$, in addition to being older, are less metal rich and more $[\alpha / \mathrm{Fe}]$ enhanced. Although Graves et al. (2010) did not present their findings in this way - their stacks contain a mix of objects from different bins in our scheme - we show in Appendix B that our results are qualitatively consistent, even though they did not account for IMF gradients whereas we do. However, there are important differences. For example, their binning scheme hides the fact that objects in our bin B10-L are extremely old. In addition, their analysis extends to much smaller $\sigma_{0}$ and $L_{r}$, in part because they do not distinguish between Es and S0s. Indeed, they did not consider the FR/SR dichotomy at all, whereas our results indicate this separation is useful.

While the FR/SR dichotomy was addressed by the ATLAS $^{3 \mathrm{D}}$ collaboration (McDermid et al. 2015), our results differ from theirs in a number of respects. Our analysis is confined to a much smaller range of $\sigma_{0}$ then theirs. However, at the highest $\sigma_{0}$ where we can compare results, they report that the vast majority of FRs are more than 10 Gyrs old. Few of our FRs are this old. As a result, while they report that smaller FRs are older, we find the opposite. Instead, our size-age scaling agrees with the age-rotation anti-correlation for early-types reported by SAMI (van de Sande et al. 2018).

We find strong metallicity gradients but weaker age and $[\alpha / \mathrm{Fe}]$ gradients, in qualitative agreement with previous work (see Introduction). In addition, the IMF gradients we find are qualitatively consistent, though slightly weaker than, the many recent IFU-based studies cited in the Introduction. However, we find that self-consistently accounting for gradients when estimating $M_{*}$ and $M_{\text {dyn }}$ yields good agreement between the two, especially for SRs, because gradients reduce the $M_{\mathrm{dyn}}$ estimate (more than they increase $\left.M_{*}\right)$. Thus, our resolution of the $M_{*}-M_{\mathrm{dyn}}$ discrepancy is opposite to that which has been advocated in the recent literature (Cappellari 2016; Li et al. 2017).

\section{CONCLUSIONS}

We used the methodology of Paper I to estimate single stellar population parameters using stacked spectra of elliptical galaxies binned in $\sigma_{0}$ and $L_{r}$ which were further subdivided based on $R_{e}$ in each bin (Figure 2) as well as on rotation (Figure 4 and Table 1). Our analysis has uncovered a number of trends. We first summarize our findings having to do with global quantities before describing gradients.

\subsection{Global quantities}

- Absorption line-strengths show clear differences between $L_{r}$ and $\sigma_{0}$ bins, and a clear dependence on size in each bin (Figure 9). The Es with larger sizes in a bin are older, less metal-rich and more $\alpha$-enhanced (Figure 12).

- Many of the trends with size are driven by the fact that Es with above average sizes for their $L_{r}$ and $\sigma_{0}$ tend to be slow rotators, whereas smaller than average Es are fast rotators (Figure 7).

- Absorption line strengths (Figures 10 and 11) and SSP inferred stellar populations show a strong dichotomy between fast and slow rotators (Figure 13), which drives many of the trends with size: FRs are younger, more metal rich, and less $\alpha$-enhanced. This confirms the age-rotation anti-correlation for early-types reported by SAMI (van de Sande et al. 2018), shows that rotation matters for the other stellar population parameters as well, and shows that these trends are present even when S0s have been removed from the early-type population. This agreement is reassuring, as few of our FRs are more than 10 Gyrs old, and this is in contrast to ATLAS ${ }^{3 \mathrm{D}}$ (see Figure 11 of McDermid et al. 2015).

- Ignoring the SR/FR dichotomy can lead to puzzling results. Although more compact Es are younger than their larger counterparts of the same mass (Figure 17), if one estimates ages from stacked spectra which ignore the SR/FR 
dichotomy, one finds that more compact galaxies are older rather than younger (Figure 18). This strongly suggests one should be cognizant of the SR/FR dichotomy when studying stellar populations and assembly histories.

- The stellar populations of S0s and fast rotating Es are similar at lower $\sigma_{0}$, consistent with the suggestion that FR Es could be misclassified S0s (Emsellem et al. 2011; Cappellari 2016). However, above $\sigma_{0} \sim 200 \mathrm{~km} \mathrm{~s}^{-1}$, S0s tend to have larger rotation $\lambda_{e}$ (Figure 4), smaller $n$ (Figure 5), smaller $\mathrm{B} / \mathrm{T}$ (Figure 6), younger ages, smaller $\alpha$-enhancements, and larger metallicities (Figures 10 and 11) than FR Es. Therefore, had we combined S0s with Es in our analyses, then this would have made the differences between FRs and SRs even more pronounced.

- The metallicity of the FRs in bin B11 is significantly higher than in other bins (Figures 10, 11 and 13). The high central metallicity and lower $[\alpha / \mathrm{Fe}]$ of FRs in this bin suggest that they may have formed from a relatively recent major merger involving at least one FR (spiral or S0) - they can not be FRs whose disks have faded or been stripped away. This is consistent with the fact that the FR population basically disappears at $M_{*} \geq 3 \times 10^{11} M_{\odot}$.

- The FRs in bin B10-L are unusual: their properties are more similar to those of slow rotators than to S0s (of the same $L_{r}$ and $\sigma_{0}$ ). The majority of these galaxies are centrals of halos less massive than $10^{13} M_{\odot}$ (Figure 21). This suggests that fast rotators at low $L_{r}$ (i.e. in bin B10) are systems with larger dissipation than their slow rotator counterparts with either more quiescent merger histories, or histories in which the assembly happened much earlier compared to the FRs at higher masses (i.e. bin B11).

- There are two distinct types of SRs. Although both are $\alpha$-enhanced, at smaller $\sigma_{0}$ and/or $L_{r}$ (bins B00, B10 and B11) SRs are old and metal poor, whereas at large $\sigma_{0}$ and $L_{r}$ (bin B21) they are not quite as old and are more metal rich (compare red/pink lines in the middle panels of Figure 13 with the solid lines in the left panels). From this it appears that the general belief that age, metallicity and $\alpha$ enhancement increase monotonically with $\sigma$ only applies if one has averaged over SRs and FRs (or over all sizes at a given $\sigma$ ).

\subsection{Gradients}

Regarding gradients, we found that:

- Gradients preserve the global correlation of $\sigma$ with age, metallicity and $M_{*} / L_{r}$ while we observe a weak local anticorrelation with $[\alpha / \mathrm{Fe}]$ (Figure 12).

- At fixed $L_{r}$, ages track the local value of velocity dispersion. When we split our sample in objects which are larger/smaller than average for their bin they define different tracks. However, the ages of each subsample (i.e. small or large sizes) of a given $L_{r}$ still track the local value of velocity dispersion (in panels which are second from top in Figure 12 dark/light blue connect to dark/light green symbols, and brown/orange connect to red/pink symbols).

- The local metallicity and IMF slope are also strongly correlated (compare top and third from top panels in Figure 12).

- The anti-correlation between metallicity and age or $\alpha$ enhancement also applies locally. This is more evident for bins B00 and B10 (e.g. in right hand panels of Figure 12 compare dark/light blue lines in the second through fourth panels).

- Inside $\sim R_{e}$ SRs are remarkably homogeneous, being uniformly old, metal poor and $\alpha$-enhanced for bins B00, B10 and B11 (right hand panels of Figure 13). SRs in bin B21 are slightly younger and more metal rich (pink and red symbols in Figure 13). In this context, it is worth noting that $\mathrm{Gu}$ et al. (2018) have reported that early-type galaxies in clusters show no correlation between $[\alpha / \mathrm{Fe}]$ and galaxy mass, nor any gradient in $[\alpha / \mathrm{Fe}]$, whereas $[\alpha / \mathrm{Fe}]$ decreases from the center towards the outskirts in field galaxies. They argue that their observations indicate a coordinated assembly of the stellar mass in cluster galaxies. However, this cannot be the full story because the majority of B00 and B10 objects in our sample do not inhabit massive clusters (Figures 20), yet they are remarkably homogeneous. Moreover, despite being in relatively isolated enviroments, they have $[\alpha / \mathrm{Fe}]$ increasing towards the outskirts. (Unfortunately, as we discuss in Paper I, $[\alpha / \mathrm{Fe}]$ gradients are model dependent.)

- Age gradients in SRs tend to be stronger in the less massive objects. Metallicity gradients are generally stronger than age gradients. While it is often argued that stronger gradients imply quiescent merger histories at least in the recent past, some recent work suggests that minor mergers tend to create positive age gradients, bring in $\alpha$-enhanced stars, and steepen metallicity gradients (Hirschmann et al. 2015). Our measurements of the B21 SRs do indeed show such gradients. On the other hand the properties of SRs with smaller $\sigma_{0}$ and/or $L_{r}$ (bins B00, B10 and B11) can be explained by either a scenario in which both star formation and assembly happened at very early times, or the assembly at later times involved minor mergers with old, low metallicity and highly $\alpha$-enhanced systems. The lower $[\mathrm{M} / \mathrm{H}]$ of SRs in bins B00, B10 and B11 imply that they could not have been formed from minor mergers of FRs having similar properties to the ones we observe (i.e. they must have been fast rotators with low $[\mathrm{M} / \mathrm{H}]$ and $[\alpha / \mathrm{Fe}])$.

- FRs are slightly less $\alpha$-enhanced in their centers, suggesting more extended star formation histories in the central regions (though we again caution that $[\alpha / \mathrm{Fe}]$ gradients are model dependent). $[\alpha / \mathrm{Fe}]$ increasing slightly towards the outskirts has also been reported by Boardman et al. (2017) in a sample of 12 intermediate mass ETGs (E+S0s with $M_{*}<2 \times 10^{11} M_{\odot}$ ), and by Martín-Navarro et al. (2018). Such gradients are in qualitative agreement with the outsidein models of Pipino et al. (2006), although more recent work suggests that the gradients imply mostly quiet evolutionary histories in which many objects have experienced some kind of gaseous interaction in recent times.

- Gradients in $M_{*} / L_{r}$ are stronger for FRs. Only SRs with large $L_{r}$ and $\sigma_{0}$ (i.e. B21) show comparable $M_{*} / L_{r}$ gradients (bottom middle and right panels of Figure 13).

- $M_{*} / L$ gradients matter when estimating the dynamical mass, confirming what we found in Paper I. Even when we subdivide each $L_{r}$ and $\sigma_{0}$ bin on the basis of half-light radius $R_{e}$ and rotation, self-consistently accounting for gradients when estimating $M_{*}$ and $M_{\mathrm{dyn}}$ yields good agreement between the two (Figure 16). This is because accounting for $M_{*} / L$ gradients reduces $M_{\text {dyn }}$ by $\sim 0.2$ dex so it agrees with the stellar population estimate of $M_{*}$ (rather than the other way around). Moreover, because only the mass in the central 
regions may have bottom-heavy IMFs (Figure 14), this $M_{*}$ is more consistent with using a Kroupa IMF than a Salpeter IMF, even for massive galaxies.

- This agreement between $M_{*}$ and $M_{\text {dyn }}$ means we no longer need to specify an IMF when specifying the mass scale at which scaling relations change and the population becomes dominated by SRs: This scale is $3 \times 10^{11} M_{\odot}$ (e.g. Figure 17).

In his review of the field Cappellari (2016) states that early-type galaxies form via two main channels: FRs start as star-forming discs and grow their bulges via dissipative processes, followed by quenching, while the more massive SRs form as in the two-phase scenario, with an early rapid dissipative formation followed by repeated dry merger events. Our results suggest that, within $\sim R_{e}$, SR Es were indeed formed in gas-rich, rapid star formation events at $z \sim 4$. This will have led to relatively steep radial metallicity variations and positive $[\alpha / \mathrm{Fe}]$ gradients which were weakened by the subsequent assembly history, in broad agreement with Martín-Navarro et al. (2018).

As most of our analysis was confined to scales smaller than the half-light radius, the next natural observational step is to push to larger scales, to see how gradients change (do they flatten out? invert?). From the theory side we are left with a number of open questions which we hope will stimulate further work. Why are FR Es so much younger than SR Es? Are FR Es just S0s seen face-on? In this case, why are the properties of FRs in bin B10-L more similar to those of slow rotators than to S0s? Are FR Es with large $L_{r}$ and $\sigma_{0}$ (i.e. in bin B11, where metallicity is significantly higher than in other bins) formed from a relatively recent major merger, perhaps involving a fast rotator galaxy (spiral or S0), while SR Es are the result of many dry minor mergers? Or did SR Es form through major dry mergers which happened at very early times? Could major versus minor dry mergers explain why there are two types of SRs, one metal rich and the other metal poor?

\section{ACKNOWLEDGEMENTS}

We are grateful to E. Emsellem for helpful correspondence about whether or not FRs are really S0s, to K. Westfall and T. Parikh for many helpful discussions about the MaNGA data, to C. Maraston, D. Thomas, A. Vazdekis and G. Worthey for correspondence about their SSP models, and to the referee for a helpful report. This work was supported in part by NSF grant AST-1816330.

Funding for the Sloan Digital Sky Survey IV has been provided by the Alfred P. Sloan Foundation, the U.S. Department of Energy Office of Science, and the Participating Institutions. SDSS acknowledges support and resources from the Center for High-Performance Computing at the University of Utah. The SDSS web site is www.sdss.org.

SDSS is managed by the Astrophysical Research Consortium for the Participating Institutions of the SDSS Collaboration including the Brazilian Participation Group, the Carnegie Institution for Science, Carnegie Mellon University, the Chilean Participation Group, the French Participation Group, Harvard-Smithsonian Center for Astrophysics, Instituto de Astrofísica de Canarias, The Johns Hopkins University, Kavli Institute for the Physics and Mathematics of the Universe (IPMU) / University of Tokyo,
Lawrence Berkeley National Laboratory, Leibniz Institut für Astrophysik Potsdam (AIP), Max-Planck-Institut für Astronomie (MPIA Heidelberg), Max-Planck-Institut für Astrophysik (MPA Garching), Max-Planck-Institut für Extraterrestrische Physik (MPE), National Astronomical Observatories of China, New Mexico State University, New York University, University of Notre Dame, Observatório Nacional / MCTI, The Ohio State University, Pennsylvania State University, Shanghai Astronomical Observatory, United Kingdom Participation Group, Universidad Nacional Autónoma de México, University of Arizona, University of Colorado Boulder, University of Oxford, University of Portsmouth, University of Utah, University of Virginia, University of Washington, University of Wisconsin, Vanderbilt University, and Yale University.

\section{REFERENCES}

Behroozi P., et al., 2015, MNRAS, 454, 3020

Bernardi M., Sheth R. K., Nichol R. C., Schneider D. P., Brinkmann J., 2005, AJ, 129, 61

Bernardi M., Nichol R. C., Sheth R. K., Miller C. J., Brinkmann J., 2006, AJ, 131, 1288

Bernardi M., Roche N., Shankar F., Sheth R. K., 2011, MNRAS, 412, L6

Bernardi M., Meert A., Sheth R. K., Vikram V., HuertasCompany M., Mei S., Shankar F., 2013, MNRAS, 436, 697

Bernardi M., Meert A., Sheth R. K., Fischer J.-L., HuertasCompany M., Maraston C., Shankar F., Vikram V., 2017a, MNRAS, 467, 2217

Bernardi M., Fischer J.-L., Sheth R. K., Meert A., HuertasCompany M., Shankar F., Vikram V., 2017b, MNRAS, 468, 2569

Bernardi M., et al., 2018a, MNRAS, 475, 757

Bernardi M., Sheth R. K., Dominguez-Sanchez H., Fischer J.-L., Chae K.-H., Huertas-Company M., Shankar F., 2018b, MNRAS, 477, 2560

Blanton M. R., et al., 2017, AJ, 154, 28

Boardman N. F., et al., 2017, MNRAS, 471, 4005

Bundy K., Fukugita M., Ellis R. S., Targett T. A., Belli S., Kodama T., 2009, ApJ, 697, 1369

Bundy K., et al., 2015, ApJ, 798, 7

Cappellari M., 2016, ARA\&A, 54, 597

Cappellari M., et al., 2011, MNRAS, 413, 813

Cappellari M., et al., 2013, MNRAS, 432, 1862

Croom S. M., et al., 2012, MNRAS, 421, 872

Davies R. L., Sadler E. M., Peletier R. F., 1993, MNRAS, 262, 650

Domínguez Sánchez H., Huertas-Company M., Bernardi M., Tuccillo D., Fischer J. L., 2018, MNRAS, 476, 3661

Domínguez Sánchez H., Bernardi M., Brownstein J. R., Drory N., Sheth R. K., 2019, arXiv e-prints, p. arXiv:1904.11992

Emsellem E., et al., 2004, MNRAS, 352, 721

Emsellem E., et al., 2007, MNRAS, 379, 401

Emsellem E., et al., 2011, MNRAS, 414, 888

Ferreras I., et al., 2019, arXiv e-prints, p. arXiv:1905.03257

Fischer J.-L., Bernardi M., Meert A., 2017, MNRAS, 467, 490

Fischer J.-L., Domínguez Sánchez H., Bernardi M., 2019, MNRAS, 483, 2057

Fraser-McKelvie A., Aragón-Salamanca A., Merrifield M., Tabor M., Bernardi M., Drory N., Parikh T., Argudo-Fernández M., 2018, MNRAS, 481, 5580

González Delgado R. M., et al., 2014, A\&A, 562, A47

González Delgado R. M., et al., 2015, A\&A, 581, A103

Graham M. T., et al., 2018, MNRAS, 477, 4711 
Graves G. J., Faber S. M., Schiavon R. P., 2010, ApJ, 721, 278 Greene J. E., Janish R., Ma C.-P., McConnell N. J., Blakeslee J. P., Thomas J., Murphy J. D., 2015, ApJ, 807, 11 Grevesse N., Sauval A. J., 1998, Space Sci. Rev., 85, 161 Gu M., Conroy C., Brammer G., 2018, ApJ, 862, L18 Hilz M., Naab T., Ostriker J. P., 2013, MNRAS, 429, 2924

Hirschmann M., Naab T., Ostriker J. P., Forbes D. A., Duc P.-A., Davé R., Oser L., Karabal E., 2015, MNRAS, 449, 528

Johansson J., Thomas D., Maraston C., 2012, MNRAS, 421, 1908

Koleva M., Prugniel P., De Rijcke S., Zeilinger W. W., 2011, MNRAS, 417, 1643

La Barbera F., Ferreras I., Vazdekis A., de la Rosa I. G., de Carvalho R. R., Trevisan M., Falcón-Barroso J., Ricciardelli E., 2013, MNRAS, 433, 3017

La Barbera F., Vazdekis A., Ferreras I., Pasquali A., Cappellari M., Martín-Navarro I., Schönebeck F., Falcón-Barroso J., 2016, MNRAS, 457, 1468

Larson R. B., 1974, MNRAS, 166, 585

Law D. R., et al., 2015, AJ, 150, 19

Li H., et al., 2017, ApJ, 838, 77

Li H., et al., 2018, MNRAS, 476, 1765

Man A. W. S., Zirm A. W., Toft S., 2016, ApJ, 830, 89

Martín-Navarro I., La Barbera F., Vazdekis A., Falcón-Barroso J., Ferreras I., 2015a, MNRAS, 447, 1033

Martín-Navarro I., La Barbera F., Vazdekis A., Ferré-Mateu A., Trujillo I., Beasley M. A., 2015b, MNRAS, 451, 1081

Martín-Navarro I., et al., 2015c, ApJ, 806, L31

Martín-Navarro I., Vazdekis A., Falcón-Barroso J., La Barbera F., Yıldırım A., van de Ven G., 2018, MNRAS, 475, 3700

McDermid R. M., et al., 2015, MNRAS, 448, 3484

Mendel J. T., Simard L., Palmer M., Ellison S. L., Patton D. R., 2014, ApJS, 210, 3

Mo H., van den Bosch F. C., White S., 2010, Galaxy Formation and Evolution

Paranjape A., Hahn O., Sheth R. K., 2018, MNRAS, 476, 5442

Parikh T., et al., 2018, MNRAS, 477, 3954

Parikh T., et al., 2019, MNRAS, 483, 3420

Pipino A., Matteucci F., Chiappini C., 2006, ApJ, 638, 739

Sánchez-Blázquez P., Forbes D. A., Strader J., Brodie J., Proctor R., 2007, MNRAS, 377, 759

Sánchez S. F., et al., 2012, A\&A, 538, A8

Sarzi M., Spiniello C., La Barbera F., Krajnović D., van den Bosch R., 2018, MNRAS, 478, 4084

Scott N., et al., 2009, MNRAS, 398, 1835

Spolaor M., Proctor R. N., Forbes D. A., Couch W. J., 2009, ApJ, 691, L138

Spolaor M., Kobayashi C., Forbes D. A., Couch W. J., Hau G. K. T., 2010, MNRAS, 408, 272

Tang B., Worthey G., 2017, MNRAS, 467, 674

Thomas D., Maraston C., Bender R., Mendes de Oliveira C., 2005, ApJ, 621, 673

Vaughan S. P., Davies R. L., Zieleniewski S., Houghton R. C. W., 2018a, MNRAS, 475, 1073

Vaughan S. P., Davies R. L., Zieleniewski S., Houghton R. C. W., 2018b, MNRAS, 479, 2443

Vazdekis A., et al., 2015, MNRAS, 449, 1177

Wake D. A., et al., 2017, AJ, 154, 86

Westfall K. B., et al., 2019, arXiv e-prints,

White S. D. M., 1980, MNRAS, 191, 1P

Yang X., Mo H. J., van den Bosch F. C., Pasquali A., Li C., Barden M., 2007, ApJ, 671, 153

Zhuang Y., Leaman R., van de Ven G., Zibetti S., Gallazzi A., Zhu L., Falcón-Barroso J., Lyubenova M., 2019, MNRAS, 483, 1862

Zibetti S., Gallazzi A. R., Hirschmann M., Consolandi G., FalcónBarroso J., van de Ven G., Lyubenova M., 2019, arXiv eprints, p. arXiv:1906.02209

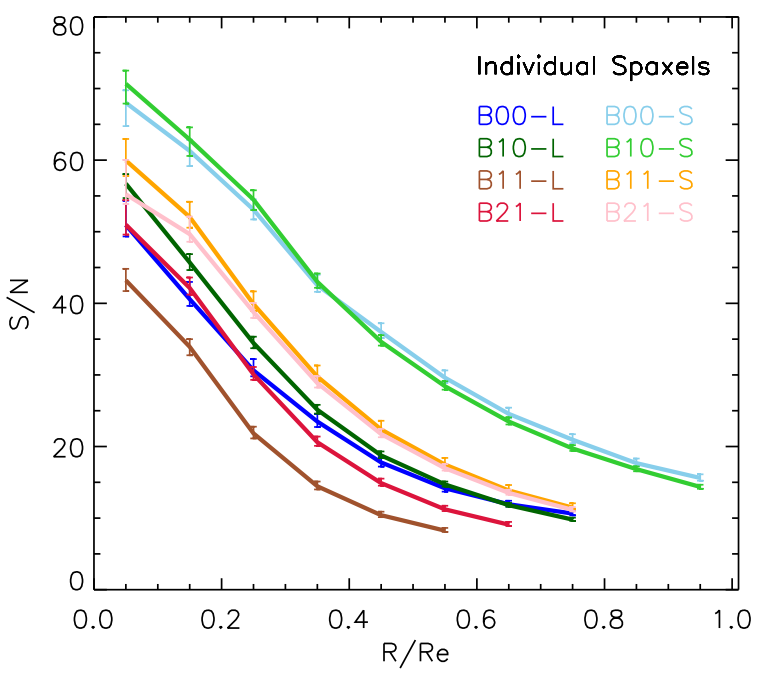

Figure A1. The median signal-to-noise per spaxel decreases monotonically with distance from the center, for the eight bins defined in Table 1. The smaller objects of each type (lighter shade of each color) have higher S/N. This represents independent confirmation from the spaxels that the photometric analysis has correctly separated each $\sigma$ and $L_{r}$ bin into high- and low-surface brightness (small and large sizes) objects. The typical S/N per spaxel is less than 100 , which is why a stacking analysis is necessary.

van Dokkum P., Conroy C., Villaume A., Brodie J., Romanowsky A. J., 2017, ApJ, 841, 68

van de Sande J., et al., 2018, Nature Astronomy, 2, 483

\section{APPENDIX A: SIGNAL-TO-NOISE OF STACKED SPECTRA}

The analysis in the main text uses stellar population synthesis models to interpret a number of absorption line strengths. It is well known that spectra with $\mathrm{S} / \mathrm{N}>100$ are required to make sufficiently reliable measurements. Here we show that our stacked spectra do indeed have sufficient signal-to-noise.

As Figure A1 shows, the typical $\mathrm{S} / \mathrm{N}$ in a spaxel lies well-below this desired value of $\mathrm{S} / \mathrm{N}=100$. (We note in passing that the $\mathrm{S} / \mathrm{N}$ is clearly larger for the more compact galaxies. This is reassuring, since the $R_{e}$ determinations were made from the photometry, without regard to the spectroscopy. As we noted, smaller $R_{e}$ implies larger surface brightness at fixed $L_{r}$, hence higher $\mathrm{S} / \mathrm{N}$ in a spaxel.)

Figure A2 shows the number of spaxels in each radial bin which contribute to our results for the eight bins in $L_{r}$, $\sigma_{0}$ and $R_{e}$ defined in Table 1 . Some of the curves decrease at large $R$ because, for the largest galaxies, the spaxels may not cover the entire region within $R_{e}$. To appreciate why, Figure A3 shows the distribution of apparent size and redshift for these Es. Since the spaxel size is fixed, for fixed $R_{e}$, nearby objects are sampled by more spaxels. Conversely, at fixed $z$, the objects with larger $R_{e}$ (darker shade of each color) are sampled by more spaxels, meaning they contribute more spaxels to a stack on the scale $R / R_{e}$. Figure A2 shows that this bias is stronger for bin B11-L. Dashed curves show scales which are compromised by incomplete IFU coverage, so they are not used when studying gradients. 


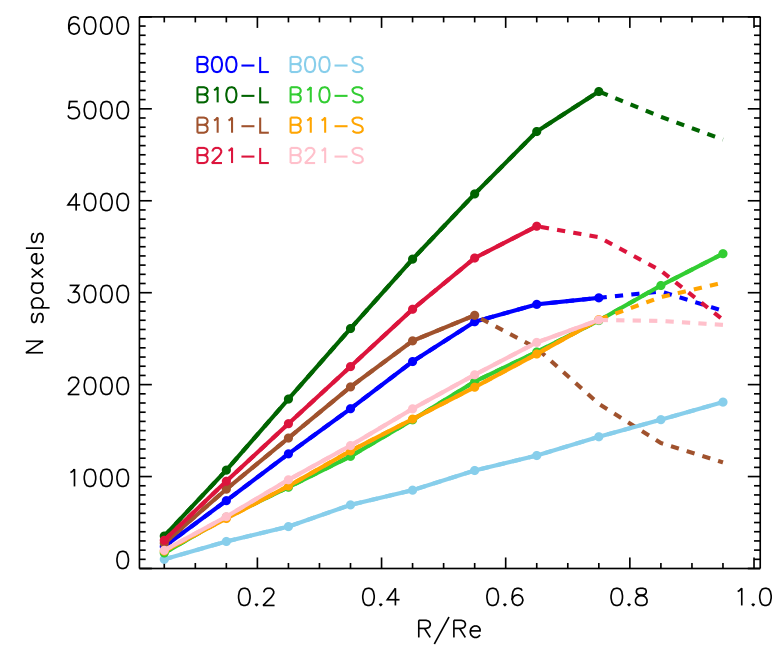

Figure A2. Number of spaxels in each radial bin which contribute to our results for the eight bins in $L_{r}, \sigma_{0}$ and $R_{e}$ defined in Table 1 (lighter shades show smaller sizes). Dashed curves show scales which are compromised by incomplete IFU coverage, so they are not used when studying gradients.

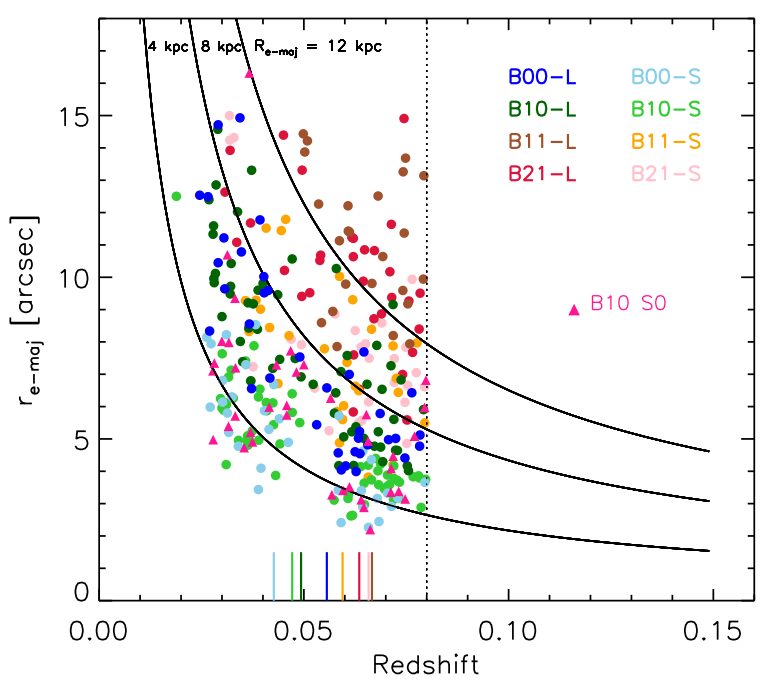

Figure A3. Apparent size - redshift relation for the objects in our sample. Objects with large apparent sizes are covered by more fibers; at fixed $R_{e}$, lower redshift objects dominate the spaxel count. Small vertical lines show the median $z$ for each bin.

Figure A4 shows that our stacks have $\mathrm{S} / \mathrm{N}>100$ on all scales we explore in this paper.

In Section 2.2 we study the difference between slow and fast rotators. This means we must further subdivide our sample, reducing the number of spaxels which contribute to each stack. Dotted lines in Figure A5 show when there are too few spaxels to make reliable Lick index measurements: these are either slowly rotating compact low luminosity B00-S galaxies (light blue), fast-rotating large low luminosity B00-L galaxies (dark blue), fast-rotating luminous galaxies from bin B21 (pink and red), and fast-rotating large B11 galaxies (brown). Dashed curves show scales which are compromised by incomplete IFU coverage, so they are not used when studying gradients.

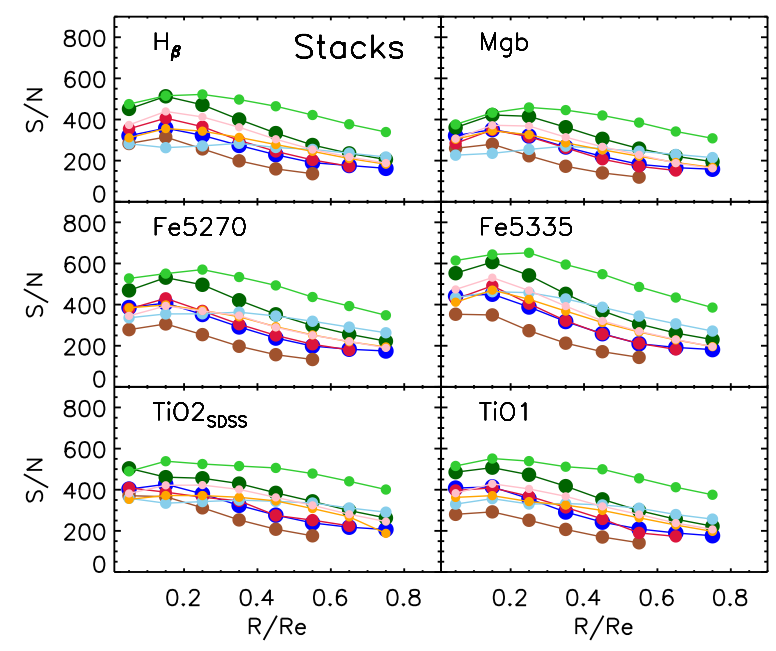

Figure A4. Signal-to-noise profiles for the Lick indices measured from the stacked spectra of galaxies in the bins defined in Table 1. Our stacks have $\mathrm{S} / \mathrm{N}>100$ on all scales we explore in this paper.

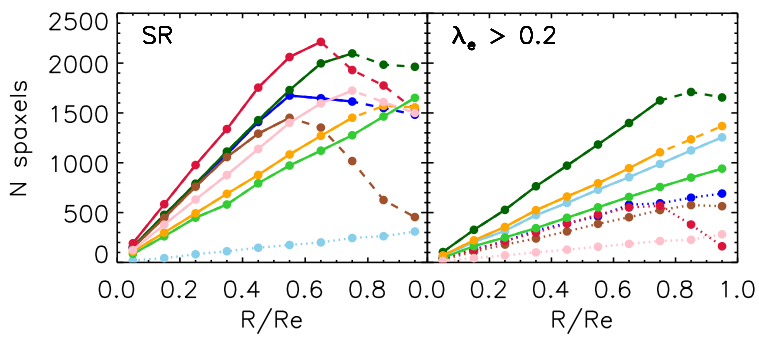

Figure A5. Number of spaxels which contribute to our slow (left) and fast rotator (right) stacks. Dotted lines connect bins in which there are too few spaxels to make reliable Lick index measurements: these are either slowly rotating compact low luminosity B00 galaxies (light blue), fast-rotating luminous galaxies from bin B21 (pink and red), or fast-rotating large B11 galaxies (brown). Dashed curves show scales which are compromised by incomplete IFU coverage, so they are not used when studying gradients.

\section{APPENDIX B: COMPARISON WITH PREVIOUS WORK}

\section{B1 Comparison with Graves et al. (2010)}

Graves et al. (2010) estimated ages, metallicities and $\alpha$ enhancements from stacked spectra of early-type galaxies in the SDSS, assuming the same (Chabrier) IMF for all objects. Converting their Table 2 scalings in $\sigma, R_{e}$ and $\Delta I_{e} \propto\left(L / R_{e}^{2}\right) R_{e}^{1.21} \sigma^{-1.16}$ into our $L, R_{e}$ and $\sigma_{0}$ yields

$$
\begin{aligned}
\text { age } & \propto R_{e}^{0.02} \sigma_{0}^{0.73} \Delta I_{e}^{-0.92} \propto R_{e}^{0.75} \sigma_{0}^{1.8} L^{-0.9} \\
{[\mathrm{Fe} / \mathrm{H}] } & \propto R_{e}^{-0.46} \sigma_{0}^{-0.47} L^{0.64} \\
{[\mathrm{Mg} / \mathrm{Fe}] } & \propto R_{e}^{0.24} \sigma_{0}^{0.58} L^{-0.27} \propto \operatorname{age}^{0.31}
\end{aligned}
$$

(if we ignore the curvature in their age relation).

Thus, they find that, at fixed $L_{r}$ and $\sigma_{0}$, objects with larger $R_{e}$ are older: a 0.1 dex difference in $R_{e}$ corresponds to an $18 \%$ change in age. This is consistent with our finding of $\mathrm{a} \sim 2$ Gyr age difference in the main text. These scalings are also consistent with our finding that, at fixed $L_{r}$ and $\sigma_{0}$, the objects with larger $R_{e}$, in addition to being older, are less metal rich and more $[\mathrm{Mg} / \mathrm{Fe}]$ enhanced. Thus, our analysis 


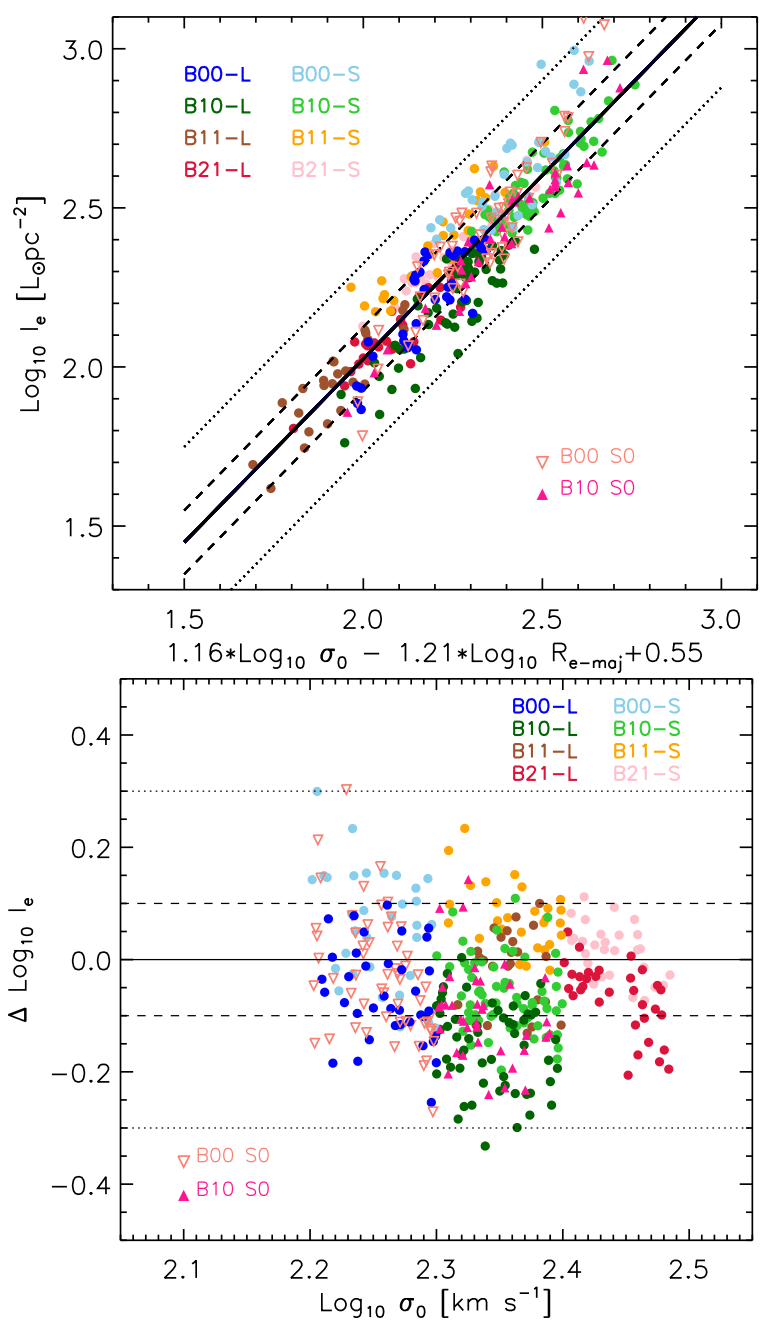

Figure B1. Top: Surface-brightness as predicted by the Fundamental Plane combination of $\sigma_{0}$ and $R_{e}$. A wide range of $L_{r}$ and $R_{e}$ have the same $I_{e}$. Bottom: Surface brightness residuals $\Delta I_{e} \propto\left(L / R_{e}^{2}\right) /\left(R_{e}^{1.21} \sigma_{0}^{-1.16}\right)$ from the Fundamental Plane vs $\sigma_{0}$. Inclusion of S0s in the B10 bin would reduce the estimated ages in that bin. Equation (B1) implies that lines of constant age slope up and to the right.

shows that these qualitative scalings persist even when one allows for IMF gradients.

Although these trends with size at fixed $\sigma_{0}$ and $L_{r}$ are in qualitative agreement with our findings, there are important differences when it comes to the scaling of, e.g., age with $\sigma_{0}$ and $L_{r}$ for Es. Figure B1 illustrates why. The colored symbols show the objects in the bins we define, plotted in the variables Graves et al. (2010) used for determining which spectra to stack. Comparison with their Figure 1 shows that their bins correspond to a horizontal division in the bottom panel of Figure B1. Their analysis extends to much smaller $\sigma_{0}$ and $L_{r}$. This is in part because they do not distinguish between Es and S0s. But over the $\sigma$ range where our samples overlap, it is clear that their stacks contain a mix of objects from different bins in our scheme. While our scheme could be crudely approximated by drawing parallelograms in this coordinate system, we believe that by working directly with $L_{r}$ and $\sigma_{0}$ our binning scheme more effectively

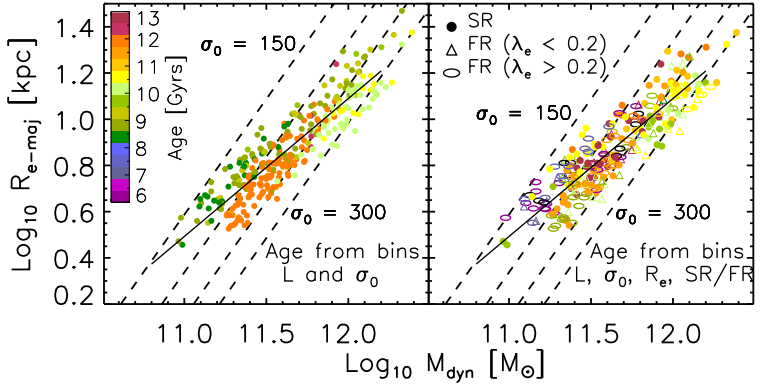

Figure B2. Same as Figure 17 of the main text, but with $M_{*}$ replaced by $4 R_{e} \sigma_{0}^{2} / G$. Dashed lines show locii of fixed $\sigma_{0}$ as labeled. In both panels, the symbol which represents a galaxy is colored by the age estimate for its bin, but the age estimates in the two panels differ. In the left hand panel, the smaller objects are older, but this does not hold in the right hand panel.

isolates objects of similar mass, so trends in it are simpler to interpret.

As a result, while we have qualitative agreement on some points - e.g. the age af bin B10-L Es, which are the oldest galaxies in our sample, have the lowest $\Delta \log _{10} I_{e}-$ there are important differences in detail. In particular, the distribution of B10 S0s in the bottom panel of Figure B1 is more similar to that of bin B10-L while their ages, metallicities and $\alpha$-enhancements are more similar to those of bin B10-S (see Figures 10 and 11). In addition, the expressions above do not accurately describe how, in the main text, age, metallicity and $[\mathrm{Mg} / \mathrm{Fe}]$ depend on $\sigma_{0}$ and $L_{r}$. E.g., their bins hide the fact that B10-L is extremely old. While the dark green symbols which show this bin do have amongst the largest values of $\Delta I_{e}$, equation (B1) would not make them significantly older than the objects in bin B21-L (dark red).

Finally, we note that our anomalous bin B10-L (dark green symbols) is rather different from the three outliers identified in Graves et al. (2010): theirs had $\sigma \leq 150 \mathrm{~km} \mathrm{~s}^{-1}$, which is lower than any of the B10 Es, and they had low $[\mathrm{Mg} / \mathrm{Fe}]$ for their otherwise normal ages. Our B10-L Es have anomalously high ages, but $[\mathrm{Mg} / \mathrm{Fe}]$, while high, is not particularly unusual given the estimated age.

\section{B2 Comparison with McDermid et al. (2015)}

The main text showed that the stellar populations of FRs and SRs are very different. The FR/SR dichotomy was not considered at all by Graves et al. (2010). However, McDermid et al. (2015) (Paper XXX of the ATLAS ${ }^{3 \mathrm{D}}$ collaboration) do study this issue. They state that compact earlytype galaxies tend to be older, more metal-rich, and more $\alpha$-enhanced than larger ETGs of the same mass. To compare directly with them, Figure B2 shows the size-dynamical mass correlation in our sample. The dashed lines shows locii of fixed $\sigma_{0}$; lines for larger $\sigma_{0}$ are displaced down and to the right. In both panels, the symbol which represents a galaxy is colored by the age estimate for its bin.

The first point to make is that their age-size trend is most dramatic when the full range of $\sigma_{0}$ is shown; our sample spans a much smaller range in $\sigma$ and $M_{\mathrm{dyn}}$ (it corresponds to the top right corner of their plot) - so the question is if the age-size correlation (at fixed mass) remains well-defined 
at the highest $\sigma_{0}$. The left hand panel suggests that, at fixed mass, the smaller objects are indeed older. While this agrees with McDermid et al. (2015), note that our most massive objects $\left(\log _{10}\left(M_{\mathrm{dyn}} / M_{\odot}\right) \geq 12\right)$ are not the oldest: objects with $\log _{10}\left(M_{\text {dyn }} / M_{\odot}\right) \sim 11.5$ are older. This is inconsistent with their Figure 6.

Moreover, their Figure 11 shows that the vast majority of FRs with $\sigma \geq 220 \mathrm{kms}^{-1}$ are more than 10 Gyrs old. As we discuss in the main text, few of our FRs are this old, and this is one reason why we find that, even at high masses, smaller objects are younger whereas McDermid et al. (2015) report the opposite.

This paper has been typeset from a $\mathrm{T}_{\mathrm{E}} \mathrm{X} / \mathrm{LAT}_{\mathrm{E}} \mathrm{X}$ file prepared by the author. 